\title{
Modelling Progressive Failure in Multi-Phase Materials Using A Phase Field Method
}

Peng Zhanga, b, Xiaofei Hu*a, b, Shangtong Yang ${ }^{\mathrm{c}}$, Weian Yao ${ }^{\mathrm{a}, \mathrm{b}}$

\author{
${ }^{a}$ State Key Laboratory of Structural Analysis for Industrial Equipment, Dalian University of \\ Technology, Dalian 116024, P. R. China \\ ${ }^{b}$ International Center for Computational Mechanics, Dalian University of Technology, Dalian, \\ 116024, P. R. China. \\ ${ }^{c}$ Department of Civil and Environmental Engineering, University of Strathclyde, Glasgow, G1 \\ IXJ, United Kingdom \\ *Corresponding author: hxf@dlut.edu.cn (Xiaofei Hu)
}

\begin{abstract}
In this paper, a new phase field method is proposed for modelling of progressive failure in multi-phase materials. Material properties of the interface between inclusion and matrix are regularized by an auxiliary interface phase field. In addition, crack initiation and propagation are simulated by using another crack phase field. Different failure mechanisms such as interface debonding, matrix cracking and the interaction between these two failure mechanisms are modelled in a unified framework. For general application of the framework, an image processing method is employed to identify the individual phases for a given multi-phase material. The proposed method is implemented into the commercial software ABAQUS through a user subroutine UEL (user defined element). The derived method is validated through an example of a single fiber reinforced composite system. Moreover, a procedure for choosing parameters of the proposed phase field model is discussed. Further, the validated method is applied to fracture analysis of a multi-phase concrete structure and complex failure mechanisms within and across the phases are captured.
\end{abstract}

Keywords: Phase field model; interface debonding; matrix cracking; multi-phase material; ABAQUS UEL

\section{Introduction}

Meso-scale modelling of progressive failure in materials with multiple phases such as concrete [1-3], fiber-reinforced composite [4-6], bones [7, 8] and braided composites [9-12] has received much attention in the past few decades. The multi-phase materials are usually characterized in terms of inclusion, matrix and interface in between. The inclusions can be arbitrary in size and shape and are distributed in the matrix. The meso-scale modelling can provide unique insight into the material fracture behavior since a number of progressive failure processes cannot otherwise be simulated, 
e.g., interface cracking/debonding [13]. In the past few decades, a few numerical approaches have been applied and widely acknowledged for modelling these complex mechanisms. Grassl et al., employed a lattice approach in simulating cracks in concrete [14]. Benkemoun et al. proposed a particular finite element meso-scale model in which a number of bar elements were employed to represent material heterogeneities and local failure [15]. In addition, different ratios of critical energy release rate and fracture strength between interface and matrix were studied [3, 4]. It has been shown that the mechanical behavior of the interface between matrix and inclusion could play an important role in the overall response of the material. In order to model the interface precisely, cohesive zone model (CZM) were widely used mainly due to the convenience for implementing the interfacial properties and accuracy in reproducing experimental results [5, 16-18]. The cohesive zone model was first proposed to overcome the unrealistic stress singularity in front of the crack tip $[19,20]$ and then implemented into finite element framework by Needleman to simulate interface debonding of the inclusion [21]. Since then, CZM has been widely applied for solving various crack propagation problems including interface debonding and crack propagation [22-24]. However, there are still some difficulties when dealing with the interactions between matrix cracking and interface debonding in a unified modelling platform [25]. For example traditional cohesive elements (CEs) along interface cannot deal with the stress transfer very well. Hence in order to model interactions between interface debonding and matrix cracking, adaptive splitting of CEs to comply complex crack configuration is needed [26, 27]. In the modelling of progressive failure of heterogeneous material, the interaction between matrix cracking and interface debonding should be well addressed. Hence, a unified numerical approach considering all the failure mechanisms and their interactions would be highly desirable.

In recent years, a variational approach of brittle fracture based on Griffith's theory [28] was proposed by Francfort and Marigo [29]. In this framework, the total energy is assumed to contain a surface term which is associated with the energy required to form a crack. The displacement field as well as the set of cracks can be obtained by minimizing the total energy. This method can overcome some limitations of Griffith's theory and linear elastic fracture mechanics (LEFM) such as inability to predict crack initiation and branching. Numerical methods dedicated to implement this framework and the Ambrosio-Tortorelli regularizations [30] are termed as phase field models of fracture [31-34]. In the phase field model, cracks are regularized by a continuous scalar phase field and crack nucleation and propagation can be simulated without additional ad-hoc criteria [3537]. Furthermore, crack tracking issues which may be very complicated for three-dimensional (3D) fracture problems are also overcome [38, 39]. Because of such advantages, phase field model is widely applied to brittle fracture problems [40-43] dynamic fracture problems [44-48], composite delamination [49] and anisotropic material fracture problems [50, 51]. Phase field model can also be applied to cohesive fracture problems. Verhoosel et al. [52] introduced a new fracture energy function to include cohesive traction-separation law in their model. And an auxiliary field along with an extra constraint was introduced to estimate crack opening displacement. However, this 
model can only be used to model crack propagation along pre-defined paths. In the study of Freddi et al. [53], they introduced a new internal length scale related energy degradation function to prevent the surface energy from being released abruptly and the cohesive laws were reconstructed through a backtracking procedure. However, the results are sensitive to the internal length scale as reported in [34]. $\mathrm{Wu}[54,55]$ proposed a unified phase field model for cohesive fracture problems, in which the parameterized degradation function and crack geometry function are used. General cohesive laws, such as bi-linear, exponential can be reproduced directly once the materials properties are known. The results are insensitive to the internal length scale as long as the crack surface is approximated precisely. However, this model is still used to model fracture problems in homogeneous material. Recently, a few phase field models are proposed for modelling the interaction between interface debonding and matrix cracking. For example, Paggi et al. [56] proposed a novel combined approach in which bulk brittle fracture is treated by phase field model and interface debonding is modelled by a compatible CE. It has been demonstrated that matrix cracking, interface debonding and the interactions between them can be modelled directly by using the proposed approach [57-59]. Nguyen et al. [60] introduced a smoothed displacement jump approximation of the interface by restoring to the level set method in phase field model. The approach allows interactions between matrix cracking and interface debonding [61, 62]. Msekh et al. [63, 64] applied the phase field approach to model progressive failure in polymer-matrix composites. More recently, Hansen-Dörr et al. [65] proposed a phase field approach for interface failure between two dissimilar materials, in which the discrete adhesive interface is regularized over a finite width. It was shown that the choice of internal length scales of crack and interface has significant impact on the prediction of crack propagation, and a reliable universal correction was proposed to improve solving accuracy.

This paper dedicates to the development of a new phase field approach for the modelling of cohesive zone model based matrix and interface failures in multi-phase materials. The material properties of the interface are regularized by using an auxiliary phase field. It results in an equivalent material field in which material properties across the interface and matrix change continuously. Moreover, another phase field is employed to model the material failure in the obtained equivalent material field. In the crack modelling the unified phase field method [54] is integrated into the present formulation for cohesive fracture. In this way, matrix cracking, interface debonding and the interaction between them can be considered concurrently. Further, the proposed method is implemented into ABAQUS through the user subroutine UEL (user defined element) [66].

\section{Regularization}

\subsection{Regularization of the interface}

It is considered that a material domain contains an inclusion, matrix and the interface between matrix and inclusion, as shown in Figure 1(a). Assuming the inclusion is much stiffer than matrix that it will keep intact during the application of stress. Therefore, a complete fracture process will 
consist of matrix cracking, interface debonding and the interaction between these two damage mechanisms. Material property discontinuity between the interface and the matrix exists, which has brought difficulties when using the phase field method. In this section, an auxiliary phase field $\eta(\boldsymbol{x})$ is introduced to regularize the material properties at interface (as shown in Figure 1(b)) to form a new material domain with continuous material properties. For this reason, $\eta(\boldsymbol{x})$ is termed as the interface phase field. By adopting the interface phase field, the material property discontinuity of matrix and interface is eliminated.

Similar to the procedure in phase field model for crack [40], the discussion begins from an infinitely expanded bar of unit cross-section with an interface at $x=0$, as shown in Figure 2(a). For this one-dimensional (1-D) problem, the interface can be described in terms of the following expressions

$$
\eta(x)= \begin{cases}1 & \text { at } x=0 \\ 0 & \text { otherwise }\end{cases}
$$

where $\eta=1$ and $\eta=0$ represent the interface and the "matrix" domain, respectively. Similar to the approximation in phase field model for crack [40], an exponential function is used to approximate the interface

$$
\eta(x)=e^{-|x| / l_{i}}
$$

where $l_{i}$ is the internal length scale of the interface phase field as shown in Figure 2(b). When $l_{i}$ is equal to zero, equation (3) will be degenerated to the discrete representation (2). It can be seen that equation (3) satisfies the following ordinary differential equation

$$
\eta(x)-l_{i}^{2} \eta^{\prime \prime}(x)=0
$$

which is subjected to boundary conditions

$$
\eta(0)=1, \quad \eta( \pm \infty)=0
$$

This ordinary differential equation is the Euler equation of the variational problem

$$
\eta=\operatorname{Arg}\left\{\inf _{\eta \in W_{i}} \Gamma_{i}(\eta)\right\}
$$

where $W_{i}=\{\eta \mid \eta(0)=1, \eta( \pm \infty)=0\}$, and the functional

$$
\Gamma_{i}(\eta)=\frac{1}{2 l_{i}} \int\left(\eta^{2}+l_{i}^{2} \eta^{\prime 2}\right) \mathrm{d} x
$$

For two-dimensional (2D) case, this functional should be extended to

$$
\Gamma_{i}(\eta)=\frac{1}{2 l_{i}} \int\left(\eta^{2}+l_{i}^{2}|\nabla \eta|^{2}\right) \mathrm{d} \Omega
$$

With the variational principle (5) and the functional (7), the Euler equation and boundary conditions of the interface phase field $\eta(\mathbf{x})$ for 2D case can be specified by 


$$
\left\{\begin{array}{cc}
\eta-l_{i}^{2} \Delta \eta=0 & \text { in } \Omega \\
\eta(\mathbf{x})=1 & \text { on } \Gamma_{i} \\
\nabla \eta \cdot \mathbf{n}=0 & \text { in } \partial \Omega
\end{array}\right.
$$

The interface phase field $\eta(\mathbf{x})$ can be solved once the location of inclusions and material properties of interface are known. $\eta(\mathbf{x})$ is used to regularize material properties of interface, and it will not be updated once it is determined at the beginning of the modelling. This is essentially different from the phase field of crack, which should be updated to reflect topology changes due to crack propagation. When the interface is smeared into the area of matrix, the equivalent material properties in the area are affected by both interface and matrix. The evaluation of equivalent material properties and more discussions on the interface phase field are presented in the following section.

\subsection{Equivalent material properties}

The equivalent material property of strength and critical energy release rate is formed from two parts. The first part is the material property of matrix in this area while the second part is the diffusive material property of interface. Here, the equivalent properties are assumed in the form of

$$
\Theta_{s}(\eta)=\Theta_{i}[1-h(\eta)]+\Theta_{m} h(\eta)
$$

where $\Theta_{s}$ represents the equivalent material properties including material strength $\sigma_{\max }^{s}$ and critical energy release rate $G_{s} . \Theta_{i}$ and $\Theta_{m}$ are the properties of interface and matrix, respectively. The function $h(\eta)$ works in the same way as the degradation function in phase field model of fracture [40]. In order to have a monotonous transition between interface and matrix, $h(\eta)$ should satisfy the following requirements

$$
h(0)=1, h(1)=0 \text { and } h^{\prime}(\eta)<0
$$

The first two requirements are used for the two critical cases

$$
\Theta_{s}(1)=\Theta_{i} \text { and } \Theta_{s}(0)=\Theta_{m}
$$

When the interface is represented discretely in the form of equation (2), the material properties are shown in Figure 3(a). In this case, the first two requirements in equation (11) are adequate. However, for the most concerned case where the interface is smeared, the last requirement in equation (11) is necessary to guarantee a monotonous material property transition as shown in Figure 3(b). Theoretically, any form of $h(\eta)$ satisfying the requirements (11) can be used in the present method. Inspired by the degradation function of phase field model of brittle fracture $[31,40$, 67], a quadratic form of $h(\eta)$ is adopted in the present study

$$
h(\eta)=(1-\eta)^{2}
$$


It can be seen that once the distribution of $\eta(\mathbf{x})$ is determined, the material properties of the mixed area can be obtained through equation (10) directly. In summary, the distributions of strength and critical energy release rate corresponding to sharp and diffusive interfaces are shown in Figure 3. It can be seen that the properties of the interface and matrix are discontinuous for a sharp interface as shown in Figure 3(a). And in Figure 3(b) where a diffusive interface approximation is introduced, the material properties are continuous with a monotonous transition.

\subsection{Regularization of the crack}

The material domain containing an inclusion can now be divided into two parts. The first part is the inclusion domain $\Omega_{i n c}$ which is the area occupied by inclusions. The inclusion domain is assumed to be intact throughout the modelling. Hence, the equivalent material property of inclusion and interface is not discussed for this area. The remaining area is termed as mixed domain $\Omega_{\text {mix }}$, it is occupied by matrix and interface. Since the interface is smeared into this domain as discussed in section 2.1, equivalent material properties should be used in this area. In this section, a phase field model is employed to regularize cracks in the mixed domain $\Omega_{\text {mix }}$. The total potential of the whole domain can be specified as

$$
W=W_{m i x}+W_{i n c}-W_{e x t}
$$

where $W_{\text {mix }}$ is the total energy containing deformation energy and fracture energy in the mixed area. $W_{\text {inc }}$ is the elastic energy of inclusion and $W_{\text {ext }}$ is the external potential.

According to the theory of the phase field model of fracture [29, 31, 40, 67], the energy potential of mixed area can be specified as follows,

$$
W_{\text {mix }}=\int_{\Omega_{\text {mix }}} \omega(d) \psi_{\varepsilon} \mathrm{d} V+\int_{\Omega_{\text {mix }}} G_{s}(\eta) \gamma(d, \nabla d) \mathrm{d} V
$$

where

$$
\gamma(d, \nabla d)=\frac{1}{c_{0}}\left(\frac{\alpha(d)}{l_{0}}+l_{0}|\nabla d|^{2}\right)
$$

is the crack surface density functional whose integral in domain $\Omega_{\text {mix }}$ represents the total crack length in $2 \mathrm{D}$ (or area in $3 \mathrm{D}$ ). $d(\mathbf{x})$ is the phase field of crack, $l_{0}$ is the internal length scale related to the width of the smeared crack. $\psi_{\varepsilon}$ is the elastic energy potential. $G_{s}$ is the equivalent critical energy release rate. The function $\alpha(d)$ is the crack geometry function [54] and $c_{0}=4 \int_{0}^{1} \sqrt{\alpha(s)} \mathrm{d} s . \omega(d)$ is the energy degradation function used to characterize the loss of material stiffness due to damage. Generally, an artificial parameter $\kappa$ with small absolute value is recommended to be added into the degradation function to improve numerical stability [68]. 
However, in the present study this parameter is set to be zero to avoid introducing artificial stiffness.

For the inclusion area the elastic bulk energy is specified as follows,

$$
W_{i n c}=\int_{\Omega_{i n c}} \psi_{i n c} \mathrm{~d} V
$$

Substituting equations (15) and (17) into (14), the total potential of the considered domain can be rewritten as

$$
\begin{gathered}
W=\int_{\Omega_{\text {mix }}} \omega(d) \psi_{\varepsilon} \mathrm{d} V+\int_{\Omega_{\text {mix }}} \frac{G_{s}(\eta)}{c_{0}}\left(\frac{\alpha(d)}{l_{0}}+l_{0}|\nabla d|^{2}\right) \mathrm{d} V \\
+\int_{\Omega_{\text {inc }}} \psi_{\text {inc }} \mathrm{d} V-\int_{\partial \Omega_{t}} \mathbf{t}^{*} \mathbf{u} \mathrm{d} S
\end{gathered}
$$

where and $\mathbf{t}^{*}$ is the vector the external loads and $\mathbf{u}$ is the vector of displacements. The variation of the above functional can be given as follows,

$$
\begin{aligned}
\delta W= & \int_{\Omega_{\text {mix }}} \omega(d) \frac{\partial \psi_{\varepsilon}}{\partial \boldsymbol{\varepsilon}} \delta \boldsymbol{\varepsilon} \mathrm{d} V+\int_{\Omega_{\text {inc }}} \frac{\partial \psi_{i n c}}{\partial \boldsymbol{\varepsilon}} \delta \boldsymbol{\varepsilon} \mathrm{d} V-\int_{\partial \Omega_{t}} \mathbf{t}^{*} \delta \mathbf{u d} S \\
& +\int_{\Omega_{\text {mix }}} \psi_{\varepsilon} \omega^{\prime}(d) \delta d \mathrm{~d} V+\int_{\Omega_{\text {mix }}} \frac{G_{s}(\eta)}{c_{0}}\left(\frac{\alpha^{\prime}(d)}{l_{0}} \delta d+2 l_{0} \nabla d \nabla \delta d\right) \mathrm{d} V
\end{aligned}
$$

Then the strong form of the problem can be obtained as

$$
\begin{cases}\omega(d) \nabla \boldsymbol{\sigma}=0 & \text { in } \Omega_{m i x} \\ \omega^{\prime}(d) \psi_{\varepsilon}+G_{s} / c_{0}\left(\alpha^{\prime}(d) / l_{0}-2 l_{0} \Delta d\right)=0 & \text { in } \Omega_{\text {mix }} \\ \nabla \boldsymbol{\sigma}=0 & \text { in } \Omega_{i n c} \\ \omega(d) \boldsymbol{\sigma} \cdot \mathbf{n}=\mathbf{t}^{*} & \text { at } \partial \Omega_{t} \\ \nabla d \cdot \mathbf{n}=0 & \text { at } \partial \Omega\end{cases}
$$

In order to prevent material cracking under compressive state, Miehe et al. [40] decomposed the strain energy density into two parts, i.e. the positive part $\psi^{e+}$ and the negative part $\psi^{e-}$. It was then followed by assuming that the energy degradation function only acted on the positive part. However, it has been shown that this remedy makes the displacement problem becoming nonlinear and increased the computational costs significantly [42]. Hence here a hybrid-formulation [42] is adopted. In this formulation only the energy density in phase field evolution equation is replaced by its positive part $\psi^{e+}$. Irrevesibility of the crack evolution is enforced by introducing a history related strain energy density $H=\max _{\tau \in[0, t]} \psi^{e+}(\varepsilon(\mathbf{x}, \tau))$ in phase field evolution euqation [40].

Since all the cracks in the present model are based on cohesive model, a unified phase field model in which various cohesive laws are integrated proposed by $\mathrm{Wu}[54,69]$ is employed. In Wu's model the material strength and cohesive softening law are implemented by a parameterized degradation function $\omega(d)$ and a polynomial crack geometric function $\alpha(d)$ which are 
specified by [54]

$$
\begin{gathered}
\omega(d)=\frac{(1-d)^{p}}{(1-d)^{p}+a_{1} d+a_{1} a_{2} d^{2}+a_{1} a_{2} a_{3} d^{3}} \\
\alpha(d)=2 d-d^{2}
\end{gathered}
$$

The values of the parameters $a_{1}, a_{2}$ and $a_{3}$ are available for various cohesive models [54].

Unlike the standard phase field model for brittle fracture [31, 40, 67], in Wu's model the damage boundedness $d \in[0,1]$ is no longer guaranteed. From the phase field evolution equation it can be seen that under very low stress levels the damage value will be negative which is unrealistic. In order to guarantee the boundedness of the phase field a modified history variable $H$ is introduced as specified by [70]

$$
H=\max _{\tau \in[0, t]}\left[\psi^{+}(\boldsymbol{\varepsilon}(\mathbf{x}, \tau)), \psi^{0}\right], \text { where } \psi^{0}=-G_{s} \frac{\alpha^{\prime}(0)}{\omega^{\prime}(0) c_{0} l_{0}}
$$

This variable is used as a critical energy under which the phase field evolution cannot be activated.

\subsection{Fracture energy conservation}

Reconsider the 1-D bar of unit cross-section with an interface as shown in Figure 5. When the interface debonding process has completed, the dissipated energy should be equal to the critical energy release rate of the interface

$$
W_{i}=G_{i}
$$

In Figure 5(a), the interface is represented discretely, and this requirement can be satisfied directly. When the crack is regularized by a crack phase field as shown in Figure 5(b), the dissipated energy can be calculated through the following equation

$$
W_{i}=\int_{-D}^{D} G_{s}(\eta) \frac{1}{c_{0}}\left(\frac{\alpha(d)}{l_{0}}+l_{0} d^{\prime 2}\right) \mathrm{d} V
$$

where $D$ is the half bandwidth of the crack phase field [54]. The fracture energy conservation gives the following relationship

$$
G_{i}=\int_{-D}^{D} G_{s}(\eta) \frac{1}{c_{0}}\left(\frac{\alpha(d)}{l_{0}}+l_{0} d^{\prime 2}\right) \mathrm{d} V
$$

In this equation, the integrand is the product of the equivalent property $G_{s}$ and the surface density functional. Integration of the latter one is equal to the area of the crack surface (which is one in this case) [54]. For a regular crack problem where $G_{s}$ is a constant, the right side of equation (26) equals to $G_{s}$. For the present study, the equivalent critical energy release rate $G_{s}$ is not a constant and it can be calculated through equation (10) as given by 


$$
G_{s}(\eta)=G_{i}[1-h(\eta)]+G_{m} h(\eta)
$$

Two different cases should be discussed separately. i.e.,

$$
G_{i}<G_{m} \text { and } G_{i} \geq G_{m}
$$

Considering that the material properties vary monotonically between interface and matrix, so for the first case we always have $G_{i}<G_{s}$. As a result, the right side of equation (26) is always greater than the left side and this equation cannot be satisfied. For the second case, equation (26) can be satisfied. In order to grantee the fracture energy conservation after regularization of the interface, special treatment should be conducted for the first case. Here we introduce a generalized critical fracture energy $\bar{G}_{i}$ to replace $G_{i}$ in the left side of equation (26). From the above discussion, it is known that $\bar{G}_{i}$ should satisfy

$$
\bar{G}_{i}<G_{i} \text { for } G_{i}<G_{m}
$$

Now, equation (26) can be rewritten as follows

$$
G_{i}=\int_{-D}^{D}\left\{\bar{G}_{i}[1-h(\eta)]+G_{m} h(\eta)\right\} \frac{1}{c_{0}}\left(\frac{\alpha(d)}{l_{0}}+l_{0}|\nabla d|^{2}\right) \mathrm{d} V
$$

where

$$
\eta=e^{-\frac{|x|}{l_{i}}} \text { and } d=1-\sin \left(\frac{|x|}{l_{0}}\right)
$$

$\bar{G}_{i}$ can be solved from equation (30) once the parameters are determined from material properties. And it should be noted that for the case $G_{i} \geq G_{m}$, equation (30) is also valid in describing the relationships among the internal length scales of interface and crack and $\bar{G}_{i}$.

In order to investigate the influence of different length scales of the crack and the interface on crack propagation and validate the obtained relationship in (30), a four-point bending specimen as shown in Figure 6 is considered. The geometric dimensions are: $L=450 \mathrm{~mm}, H=100 \mathrm{~mm}$ and $b=45 \mathrm{~mm}$. There is a pre-placed crack with length $a=50 \mathrm{~mm}$ existing in the beam along the interface as denoted by the red dotted line in the figure. The material properties are specified as follows: Young's modulus $E=2.0 \times 10^{4} \mathrm{MPa}$, Poisson's ratio $v=0.2$. The critical energy release rates for the beam and the interface are $G_{m}=0.226 \mathrm{~N} / \mathrm{mm}$ and $G_{i}=0.113 \mathrm{~N} / \mathrm{mm}$. The internal length scale for crack is set to be $l_{0}=2.5 \mathrm{~mm}$. A mesh size $h_{e}=0.5 \mathrm{~mm}$ is adopted in the simulation. Figure 7 depicts the deformation and crack pattern when $\bar{u}=-1.2 \mathrm{~mm}$ for $l_{0} / l_{i}=1.0$. The effective crack length obtained by the integral of the crack surface density functional (16) is $50.3 \mathrm{~mm}$. 
Four different values of $l_{i}$ satisfying $l_{0} / l_{i}=0.5, l_{0} / l_{i}=1.0, l_{0} / l_{i}=1.25$ and $l_{0} / l_{i}=1.5$ are used in the simulation. With the numerical results, the total dissipated energy $W_{\text {inf }}$ by forming new crack surfaces is calculated by (25). Then, an effective critical energy release rate $G_{i, e f f}$ can be calculated by dividing $W_{i}$ by the effective crack legnth. The numerical results of $G_{i, \text { eff }}$ are depicted in Figure 8, in which the green dashed line represents the theoretical value $G_{i, \text { eff }}=G_{i}$. In order to verify the proposed theory in (30), two numerical schemes are used. The first one uses the unchanged $G_{i}$ and the second one uses the evaluated $\bar{G}_{i}$ by (30) with the equivalent material property obtained in (27). Numerical results of these two schemes are also shown in Figure 8. It can be seen that the numerical results is unstable for the first scheme, i.e. the prediction of $G_{i, e f f}$ increases with the decreasing of $l_{i}$. Similar phenomenon is reported in [65] in which the fracture energy $W_{i}$ increases with the decreasing of $l_{i}$. According to the discussions of the $1 \mathrm{D}$ case (Figure 5), it is known that this phenomenon is because that smaller $l_{i}$ introduces higher influence from the bulk material to the equivalent material property. Numerical results of the second scheme is much more stable that numerical results change slightly with the changing of $l_{i}$. However, none of the results of the two schemes matches with the theoretical value. In fact, this is an open issue of phase field method as reported in [67], the predefined critical energy release rate cannot be recovered numerically because of the effect of internal length scale and mesh size. Alternatively, a numerical effective critical energy release rate [67] $G_{i, e f f}^{\text {num }}$ specified by

$$
G_{i, e f f}^{n u m}=G_{i}\left(1+\frac{h_{e}}{1+c_{0} l_{0}}\right)
$$

is used to verify the model. The value of $G_{i, e f f}^{n u m}$ is also shown in Figure 8 by the red line, and it can be seen that the results of the second scheme match very well with this theoretical value indicating the validity of the proposed theory in (30).

From this simple problem, it can be seen that the value of $l_{0} / l_{i}$ indeed has a significant influence on the crack propagating if the original interface critical energy release rate is used in (27). And the proposed modified $\bar{G}_{i}$ in (30) works very well with the considered problem. It is noteworthy that in the recently wrok of Hansen-Dörr et al. [65], similar problem is also discussed systematically. And a similar modification of $G_{i}$ is proposed for 1D case while a further modification for $2 \mathrm{D}$ case is carried out. Unlike the present way of regularization of the interface, in their work the critical energy release in the smeared interface strip is uniform.

2.5. Discussion on the choice of parameters 
There are three parameters including two internal length scales, i.e. $l_{i}$ and $l_{0}$, and a generalized critical energy release rate $\bar{G}_{i}$. The internal length scale $l_{0}$ controls the size of the diffusive zone of crack. The value of $l_{0}$ is recommended to satisfy $l_{0}>2 h_{e}$ where $h_{e}$ is the characteristic length of element [40]. In [54], the value is recommended to be

$$
l_{0} \geq 5 h_{e}
$$

because a different degradation function is used in the phase field model. In this study, this value $l_{0} \geq 5 h_{e}$ is chosen because the phase field model of [54] is adopted herein. There are also other ways for choosing $l_{0}[39,43,71,72]$. In these studies, $l_{0}$ is fixed by relating to material properties. The explicit expression of $l_{0}$ represented by material's Young's modulus, energy release rate and tensile strength is derived. In fact, for the interface phase field $l_{i}$ plays a similar role as $l_{0}$ in the crack phase field. In the modelling of interface debonding, both of the material properties and the crack are regularized. Hence the ratio between $l_{i}$ and $l_{0}$ may affect the modelling results. In the numerical example, the choice of $l_{i}$ will be discussed, it is recommended to use $l_{i}=l_{0}$ according to the numerical results. At last, $\bar{G}_{i}$ can be solved from equation (30) once $l_{i}$ and $l_{0}$ are known. Analytical solution cannot be obtained due to mathematical complexity, but this equation can be solved numerically.

In summary, $l_{0}$ is a parameter which has been discussed extensively in the literatures: the choice of this parameter depends on the choice of the phase field model. The value of $l_{i}$ should be related to $l_{0}$, and a recommended value is given based on numerical studies. From equation (30), it can be seen that $\bar{G}_{i}$ depends on both material property $G_{i}$ and model parameters $l_{i}$ and $l_{0}$

\section{Finite element formulation}

In this section finite element formulation of the proposed phase field model is derived. In the framework of finite element method (FEM), different fields $\mathbf{u}, d$ and $\eta$ can be approximated in terms of the corresponding nodal value vectors

$$
\mathbf{u}=\mathbf{N}^{\mathrm{u}} \mathbf{u}^{e}, \quad d=\mathbf{N}^{d} \mathbf{d}^{e}, \quad \eta=\mathbf{N}^{\eta} \boldsymbol{\eta}^{e}
$$

where $\mathbf{N}^{\alpha}(\alpha=u, d, \eta)$ is the shape function matrix of field $\alpha . \mathbf{u}^{e}, \mathbf{d}^{e}$ and $\boldsymbol{\eta}^{e}$ are vectors of nodal values of an element. Then the gradient of those fields can be specified as

$$
\boldsymbol{\varepsilon}=\mathbf{B}^{u} \mathbf{u}^{e}, \nabla d=\mathbf{B}^{d} \mathbf{d}^{e}, \nabla \eta=\mathbf{B}^{\eta} \boldsymbol{\eta}^{e}
$$


where $\mathbf{B}^{\alpha}$ is a matrix of shape function derivatives for field $\alpha$. Although not necessary, identical shape function matrices are used for all the fields in the present study.

The present method is implemented into ABAQUS through UEL [66]. The nonlinear system is solved by using Newton-Raphson iteration algorithm. Four nodal degrees of freedom (DOFs) are used for each node for two displacement components and the two phase fields ( $d$ and $\eta$ ). In solving the nonlinear system of equations through UEL, the right side hand vector (RHS) and tangential stiffness matrix (AMATRX) should be defined. For interface regularization, the AMATRX $\mathbf{A}_{\eta}$ and RHS $\mathbf{S}_{\eta}$ can be obtained directly through the variation of equation (6) as specified by

$$
\begin{gathered}
\mathbf{A}_{\eta}=\int_{\Omega}\left[\frac{1}{l_{i}}\left[\mathbf{N}^{\eta}\right]^{\mathrm{T}} \mathbf{N}^{\eta}+l_{i}\left[\mathbf{B}^{\eta}\right]^{\mathrm{T}} \mathbf{B}^{\eta}\right] \mathrm{d} V \\
\mathbf{S}_{\eta}=-\int_{\Omega} \frac{1}{l_{i}} \eta\left[\mathbf{N}^{\eta}\right]^{\mathrm{T}}+l_{i}\left[\mathbf{B}^{\eta}\right]^{\mathrm{T}} \nabla \eta \mathrm{d} V
\end{gathered}
$$

According to above discussions, it is known that the interface phase field $\eta(\mathbf{x})$ will not evolve throughout the modelling. Hence, $\mathbf{A}_{\eta}$ will not be updated and $\mathbf{S}_{\eta}$ will always be zero after the first step. It is also seen that the distribution of $\eta(\mathbf{x})$ should be calculated just in the first step, no extra computational effort has to be paid in the rest of the simulation.

The displacement field and the phase field for crack $d(\mathbf{x})$ are coupled, and the AMATRX $\mathbf{A}_{u}$ and RHS $\mathbf{S}_{u}$ for displacement field can be obtained by taking variation of equation (19)

$$
\begin{gathered}
\mathbf{A}_{u}= \begin{cases}\int_{\Omega_{\text {mix }}} \omega(d)\left[\mathbf{B}^{u}\right]^{\mathrm{T}} \mathbf{D} \mathbf{B}^{u} \mathrm{~d} V & \text { For mixed domain } \\
\int_{\Omega_{\text {inc }}}\left[\mathbf{B}^{u}\right]^{\mathrm{T}} \mathbf{D B}^{u} \mathrm{~d} V & \text { For inclusion domain }\end{cases} \\
\mathbf{S}_{u}= \begin{cases}-\int_{\Omega_{\text {mix }}} \omega(d)\left[\mathbf{B}^{u}\right]^{\mathrm{T}} \mathbf{D} \mathbf{B}^{u} \mathbf{u} \mathrm{d} V & \text { For mixed domain } \\
-\int_{\Omega_{\text {inc }}}\left[\mathbf{B}^{u}\right]^{\mathrm{T}} \mathbf{D} \mathbf{B}^{u} \mathbf{u} \mathrm{d} V & \text { For inclusion domain }\end{cases}
\end{gathered}
$$

where $\mathbf{D}$ is the material stiffness matrix. It is noteworthy that the FEM formulation for linear elasticity is used in the inclusion domain, this is based on the assumption that no material damage will take place in this domain. For the phase field for crack $d(\mathbf{x})$, the AMATRX $\mathbf{A}_{d}$ and RHS $\mathbf{S}_{d}$ are also obtained by taking variation of equation (19), as specified by

$$
\begin{aligned}
\mathbf{A}_{d}= & \int_{\Omega_{\text {mix }}} \omega^{\prime \prime}(d) H\left[\mathbf{N}^{d}\right]^{\mathrm{T}} \mathbf{N}^{d}+ \\
& G_{s}(\eta, \mathbf{x})\left[\frac{1}{l_{0}}\left[\mathbf{N}^{d}\right]^{\mathrm{T}} \mathbf{N}^{d}+l_{0}\left[\mathbf{B}^{d}\right]^{\mathrm{T}} \mathbf{B}^{d}\right] \mathrm{d} V
\end{aligned}
$$




$$
\mathbf{S}_{d}=-\int_{\Omega_{\text {mix }}} H \omega^{\prime}(d)\left[\mathbf{N}^{d}\right]^{\mathrm{T}} \mathrm{d} V-\int_{\Omega_{\text {mix }}} \frac{1}{l_{0}} G_{s}(\eta)\left(d\left[\mathbf{N}^{d}\right]^{\mathrm{T}}+l_{0}^{2}\left[\mathbf{B}^{d}\right]^{\mathrm{T}} \nabla d\right) \mathrm{d} V
$$

It should be noted that in the present study a staggered scheme [41] is used hence at one iteration step the displacement problem and the phase field problem are solved separately. In [70], the way to implement staggered scheme into ABAQUS is introduced. Also the default convergence criterion in ABAQUS/Standard [66] is employed in the present paper

$$
r_{\max }^{\alpha} \leq 5 \times 10^{-3} \tilde{q}^{\alpha} \text { and } c_{\max }^{\alpha} \leq 10^{-2} \Delta u_{\max }^{\alpha}
$$

where $r_{\max }^{\alpha}(\alpha=\mathbf{u}, d$, or $\eta)$ is the maximal residual force of field $\alpha$. The vector $\tilde{q}^{\alpha}$ is the time average flux value of field $\alpha$ within the current loading increment. $c_{\max }^{\alpha}$ is the maximal solution correction of $\alpha . \Delta u_{\max }^{\alpha}$ is the largest change of the solution in the current loading increment.

\section{Image processing and finite element mesh}

\subsection{Identification of different phases}

Normally, the finite element mesh should be matched to the geometric configuration in a mesoscale model [13] due to the material property discontinuity (also known as weak discontinuity[6, 73]). This is time consuming in pre-processing when dealing with multiple inclusions. For realistic meso-scale structures, image processing method is usually used [3, 60]. However, available information on the operation details in the literature is limited. Therefore in this section the overall procedures as well as some special cases are discussed.

A squared structure with an inclusion and several voids are shown in Figure 9. Matrix, inclusion and void pixels are represented by grey, black and white quadrangles. This structure can be modelled by using regular mesh. For the elements with part of the surface being occupied by inclusion or void, they can be assumed to be fulfilled by inclusion or void. The location of a pixel can be defined by the coordinates of its centroid which are given as follows,

$$
\left\{\begin{array}{l}
x=(i-0.5) \frac{L}{N_{\max }} \\
y=(j-0.5) \frac{L}{N_{\max }}
\end{array}\right.
$$

where $i$ and $j$ represent that the pixel is in the $i$ th column and $j$ th row of the image.

\subsection{Activation of nodal degree of freedom}

There are only three types of element, i.e., inclusion element, matrix element and void element. For inclusion elements, the crack phase field $d(\mathbf{x})$ is deactivated to prevent any material damage or failure. For void elements, all the DOFs are deactivated. For matrix elements, all the DOFs are 
activated.

\subsection{Boundary conditions}

There are three fields in the proposed model i.e., displacement field, crack phase field and interface phase field. Boundary conditions for each field should be applied properly on its own domain. Special attention should be paid to $\eta(\mathbf{x})$, in which the nodes on interface (termed as interface nodes hereinafter) should be applied boundary conditions according to Equation (9). When image processing method is used, the nodes on interface should be picked out as shown in Figure 10. A simple method is introduced to find out the interface nodes. Firstly, assign the inclusion and matrix elements as 1 and 0 , respectively. For each node, find all the elements connecting to it and sum these elements' assigned values

$$
S=\sum_{i}^{m} a_{i}
$$

where $a_{i}$ is the assigned value of an element and $m$ is the total number of the elements connecting to the considered node. In the present study, only regular mesh is considered, so $m$ is either 2 for a boundary node or 4 for an internal node. The considered node is an interface node if

$$
\begin{cases}S=1, & \text { if } m=2 \\ S<4, & \text { if } m=4\end{cases}
$$

It may be noted that a similar method has also been used in [60]. Once the interface nodes are identified, the nodal value of $\eta$ on these nodes should be set to 1 according to equation (9).

\section{Numerical examples}

\subsection{Single fiber system under transverse tension}

Progressive failure process of a squared matrix domain with a single fiber is modelled. The geometry and mechanical boundary conditions are illustrated in Figure 11. Material properties are specified as follows. The matrix Young's modulus $E_{m}=4 \mathrm{GPa}$, Poisson's ratio $v_{m}=0.4$, critical energy release rate $G_{m}=250 \mathrm{~N} / \mathrm{m}$ and material strength $\sigma_{m}^{\max }=30 \mathrm{MPa}$, For the fiber, Young's modulus $E_{i n c}=40 \mathrm{GPa}$ and Poisson's ratio $v_{i n c}=0.33$. For the interface, the critical energy release rate $G_{i}=50 \mathrm{~N} / \mathrm{m}$ and the material strength $\sigma_{i}^{\max }=10 \mathrm{MPa}$. The same problem has also been studied in [74-76], and all the above material properties are obtained from them.

Experimental observations of the failure patterns [77] are shown in Figure 12. According to [77] it is noted that the crack nucleates at the interface and then propagates until reaching a critical debonding angle, and migrates into the matrix at this point. And the final crack pattern observed in the experimental test is shown in Figure 12(d). Analytical expression of the semi-debonding angle $\theta$ is available and can be specified by [77] 


$$
\theta=-2 \operatorname{sgn}\left(\beta_{D}\right) \operatorname{arcos} \sqrt{\frac{2+\left|\beta_{D}\right|}{3+\left|\beta_{D}\right|}}, \text { for } \beta_{D} \neq 0
$$

where

$$
\beta_{D}=\frac{1}{2} \frac{\left(1-2 v_{2}\right) / \mu_{2}-\left(1-2 v_{1}\right) / \mu_{1}}{\left(1-v_{2}\right) / \mu_{2}+\left(1-v_{1}\right) / \mu_{1}}
$$

with the shear modules $\mu_{i}=E_{i} / 2\left(1+v_{i}\right), i=1,2$. Hence, the semi-debonding angle for the considered material in this example is $\theta=68.89^{\circ}$.

An image containing $789 \times 789$ pixels is generated for the discussed model as shown in Figure 13. A regular mesh of $200 \times 200$ quadrilateral elements (mesh A) is used, and the interface nodes recognized by the image processing method are also shown in Figure 13.

According to [54], the crack phase field length scale $l_{0}$ should be bigger than $5 h_{e}$ where $h_{e}$ is the element size. In this simulation $h_{e}=0.005 \mathrm{~mm}$, so in order to obtained a sufficiently small smeared crack width $l_{0}=0.025 \mathrm{~mm}$ is adopted. In order to investigate the influence of the internal length scale $l_{i}$, four different cases are considered: $l_{0} / l_{i}=0.4,0.7,1.0$ and 1.2 . Meanwhile, the corresponding generalized interfacial critical energy release rates can be calculated with the known internal length scales, and given as $\bar{G}_{i}=42.2 \mathrm{~N} / \mathrm{m}, 35.7 \mathrm{~N} / \mathrm{m}, 13.5 \mathrm{~N} / \mathrm{m}$ and $1.3 \mathrm{~N} / \mathrm{m}$. It is seen that $\bar{G}_{i}$ decreases with the increasing of the value of $l_{0} / l_{i}$. In order to guarantee that $\bar{G}_{i}$ is positive, the upper limit of the ratio is $l_{0} / l_{i}=1.2$ which is evaluated through a trial-and-error analysis. Linear softening cohesive model is adopted.

The distribution of interface phase field is shown in Figure 14. It is observed that the diffusive interface concentrates in the nearby area of the interface, and attenuates rapidly. The predicted crack patterns with $l_{0} / l_{i}=1$ are shown in Figure 15. For the other three cases, the crack patterns are almost identical with this one because the values of $l_{0}$ are the same for all the cases. From the results it observed that the crack nucleates from the interface and then kinks into the matrix when reaching a certain point. The numerical predictions agree very well with the experimental observations [77]. Also the predicted semi-debonding angles are $\theta=63.0^{\circ}, 67.6^{\circ}, 68.0^{\circ}$ and $68.7^{\circ}$ respectively for $l_{0} / l_{i}=0.4,0.7,1.0$ and 1.2 . Figure 16 is the crack pattern reported in [76] by using the discontinuous approach. It can be seen that the crack pattern predicted by the present method depicted in Figure 15 is in good agreement with this result. The load-displacement curves for the four different length scale ratios are depicted in Figure 17. The numerical result reported by Nguyen et al. [76] and Labanda et al. [75] are also provided in the figure. It can be seen that the predicted peak value of the curve decreases with the increasing of the ratio $l_{0} / l_{i}$. The 
result obtained when $l_{0} / l_{i}=1$ is closest to the references. Hence, this value is used in the following simulations.

In order to investigate the effect of $l_{0}$ on the numerical results, three different cases are considered, i.e. $l_{0}=0.025 \mathrm{~mm}, 0.035 \mathrm{~mm}$ and $0.05 \mathrm{~mm} . l_{i}=l_{0}$ is used and $\bar{G}_{i}$ is calculated for each case. The predicted crack patterns for different $l_{0}$ are shown in Figure 18. The diffusive zone size increases with the increasing of the value of $l_{0}$. The predicted semi-debonding angles are $\theta=68^{\circ}, 68.2^{\circ}$ and $68.3^{\circ}$ for each case, and all the predictions have a good agreement with the analytical solution $\theta=68.89^{\circ}$. The load-displacement curves are depicted in Figure 19. All the curves coincide with each other and the reference result.

A finer mesh of $334 \times 334$ quadrilateral elements (mesh B) is employed. In mesh B the value of $l_{0}$ can be smaller since the element size is smaller than mesh A. Three cases with sufficiently small internal length scales, i.e. $l_{0}=0.015 \mathrm{~mm}, 0.018 \mathrm{~mm}, 0.022 \mathrm{~mm}$ are considered. The predicted load-displacement curves are depicted in Figure 20. It can be seen that $l_{0}$ has little effect on the predicted load-displacement curve when it is sufficiently small. Moreover it can be observed that all the curves are matching very well with each other and the results with $l_{0}=0.025 \mathrm{~mm}$ (mesh A). The predicted semi-debonding angles for these cases are $\theta=67.4^{0}, 67.5^{\circ}$ and $68^{\circ}$ which are in good agreement with the analytical solution $\theta=68.89^{\circ}$. All these results indicate that the proposed model is reasonable and reliable predictions can be obtained with fine meshes. The total CPU times were 445 minutes and 140 minutes for mesh $\mathrm{A}\left(l_{0}=0.015 \mathrm{~mm}\right)$ and mesh B $\left(l_{0}=0.025 \mathrm{~mm}\right)$, respectively. All the simulations were carried out by using a workstation with Intel Xeon E5-2620 v4 CPU@2.10 GHz processor and 128G RAM.

In summary, the present simulation has considered a single fiber system subjected to tensile loading. As the image processing method is introduced, this heterogeneous material problem can be modelled in a grid mesh. In order to investigate the influence of the internal length scales of the crack and the interface phase fields, a parametric study with four different values is conducted. All the obtained crack patterns as well as the semi-debonding angle are in good agreement with the existing numerical [76] and analytical [77] results. As for load-displacement curve, the results in Figure 17 have shown that the internal length scales have significant influence on it. And through the comparison with the numerical results reported in $[75,76]$, it is recommended that $l_{0} / l_{i}=1$ is optimal. Further, an additional parametric study with five different internal length scales is reconsidered to validate the recommendation of $l_{i}$. It is shown that both the predicted crack patterns and the load-displacement curves agree very well with the existing results if $l_{0} / l_{i}=1$ is used. 


\subsection{A concrete plate subjected to tensile loading}

In this example, a concrete plate as shown in Figure 21 is considered. The size and boundary conditions are also illustrated in the figure. This plate has also been studied in [3], from which the material properties are obtained. For the matrix which represent mortar, the following material properties are used: Young's modulus $E_{m}=25 \mathrm{GPa}$, Poisson's ratio $v_{m}=0.2$, critical energy release rate $G_{m}=60 \mathrm{~N} / \mathrm{m}$ and material strength $\sigma_{m}^{\max }=6 \mathrm{MPa}$. For the inclusion which represents aggregate, the material properties are: Young's modulus $E_{i n c}=70 \mathrm{GPa}$ and Poisson's ratio $v_{i n c}=0.2$. For the interface, the critical energy release rate is $G_{i}=30 \mathrm{~N} / \mathrm{m}$ and the material strength is $\sigma_{i}^{\max }=3 \mathrm{MPa}$. External loading is applied by displacement control through 50 load increments with a fixed step value $\Delta u=1.2 \times 10^{-3} \mathrm{~mm}$.

A regular mesh of $800 \times 800$ quadrilateral elements is used. As shown in Figure 22, void and matrix elements as well as the interface nodes are recognized through the image containing $809 \times 809$ pixels of the considered plate. Based on the previous studies, the internal length scales are approximately chosen to be $l_{0}=l_{i}=0.2325 \mathrm{~mm}$. Then the generalized interfacial critical energy release rate can be calculated through equation (30). For the present case $\bar{G}_{i}=24.5 \mathrm{~N} / \mathrm{m}$. Linear softening cohesive law is adopted. The predicted distribution of the interface phase field is shown in Figure 23. Comparing with the single fiber system, it is much more complicated due to material heterogeneities.

The predicted stress-displacement curve is shown in Figure 24, in which the numerical results of [3] and [1] are provided for comparison. The stress is the average stress calculated by dividing the total nodal forces of all the nodes on the right boundary by the plate length. The external loading increases in the beginning until reaching point A (marked in Figure 24). The predicted average stresses agree well with the numerical results reported in $[1,3]$ in which cohesive elements were placed at every element edges. The pattern of the crack phase field at point A is shown in Figure 25(a). At this stage, no crack has been formed but material damage starts to initiate from some of the interfaces. With the increasing of the external loading, cracks are initiated in the form of interface debonding and then migrate into the matrix in the form of matrix cracks. Subsequently, more interface cracks and matrix cracks are formed in the direction perpendicular to the load path and these cracks are then interconnected. The average stress decreases rapidly until reaching point B. The pattern of the crack phase field at point B is shown in Figure 25(b). The cracks continue to propagate and merge into a few dominated ones as shown in the figure. It is interesting to find that the dominated cracks are pulled by the voids and eventually pass through them, and this is actually due to stress concentration. At point $\mathrm{B}$, the load has decreased to $25 \%$ of the peak value and it continues to decrease slowly after this point. The predicted load-displacement curve between points $\mathrm{B}$ and $\mathrm{C}$ has the same trend with $[1,3]$. In this period, the cracks continue to propagate but crack 
merging is not observed. The pattern of the crack phase field at point $C$ is shown in Figure 25(c), the dominated cracks eventually merge with each other and the plate is torn apart.

A parametric study on the internal length scale is performed by adopting three additional internal length scales $l_{0}=0.30 \mathrm{~mm}, 0.40 \mathrm{~mm}$ and $0.50 \mathrm{~mm}$. The obtained load-displacement curves are depicted in Figure 24. It can be seen that the predictions with $l_{0}$ smaller than $0.40 \mathrm{~mm}$ are identical to each other. Also from the figure it can be seen that with increasing of $l_{0}$, i.e. $l_{0}=0.40 \mathrm{~mm}$ and $0.50 \mathrm{~mm}$, the global response from A to B become more brittle. This underestimation is mainly because the textural details of the digital image especially the shape of the interfaces are lost. This problem does not exist for smaller internal length scales e.g. $l_{0}=0.2325 \mathrm{~mm}$ and $0.30 \mathrm{~mm}$. The total iteration steps as well as the time cost in the modelling with different $l_{0}$ are depicted in Figure 26. It is interesting to find that the computational costs with different length scales are different by using the same finite element mesh. The computational expense decreases with the increasing of the value of $l_{0}$ despite there exist some changes of numerical predictions. The total iteration step as well as the computational time have the highest values when $l_{0}=0.2325 \mathrm{~mm}$, and they are reduced by $55.4 \%$ and $55.1 \%$ respectively when $l_{0}=0.50 \mathrm{~mm}$. The iteration steps at each load increment for different internal length scales are depicted Figure 27. It can be seen that the iteration steps have one or more peak values at certain load increments; for example, there are 212 iteration steps at the 5th load increment when $l_{0}=0.2325 \mathrm{~mm}$. When $l_{0}=0.50 \mathrm{~mm}$, the peak values are much smaller, i.e. 134 steps at the 7 th load increment. It may be an effective way to reduce the computational cost by using larger values of the internal length scale. Considering that relatively large internal length scales will result in a loss of textural details of digital image and subsequent underestimation of global mechanical response. Hence the value of $l_{0}$ should still be kept small enough, e.g., $0.4 \mathrm{~mm}$ for the present study.

In summary, the present simulation has considered a concrete plate subjected to tensile loading. The predicted maximal average stresses agree well with the numerical results reported in $[1,3]$ in which cohesive elements were placed at every element edges. The crack pattern predicted by the proposed method shows that the crack prefers to initiate from the interfaces and voids then connect to each other through matrix cracks. Multiple main cracks could present in the concrete plate. Numerical results have shown that a relatively large $l_{0}$ will lead to underestimation of the reaction stress, since improper usage of too large $l_{0}$ will lead to lose of textural details of very small aggregates such as the shape of the interface. Through the investigation of the iteration steps, it is found that a small $l_{0}$ will lead to increase of computational expense and even convergence issue. 


\section{Conclusions}

In this paper, a new approach with two phase fields has been developed and implemented for modelling the progressive failure of multi-phase materials considering matrix cracking, interface debonding and the interaction between them. An interface phase field is used to regularize the material properties of the interface and a crack phase field is used for regularizing the crack. The value of internal length scale of the interface phase field is recommended to be kept the same as the crack phase field. Based on the principle of fracture energy conservation, there exist only one artificial parameter of the proposed phase field model, which can be chosen according to the provided guidance. Moreover, cohesive crack model is employed to simulate the cracks and the proposed method is implemented into finite element software package ABAQUS through the user subroutine UEL.I It is found that a small internal length scale will increase the computational expanse and even lead to convergence issue, hence a reasonable higher value of the internal length scale is recommended in the modelling. Meanwhile, the limitation of this method has also been discussed. The proposed model is validated and it is proven that the complicated failure processes in heterogeneous material can be modelled in a unified framework.

\section{Acknowledgements}

This work was supported by the National Natural Science Foundation of China (Nos.11372065, 11502045), and the National Key Research and Development Program of China [No.2016YFB0200702].

\section{References}

[1] Lopez CM, Carol I, Aguado A. Meso-structural study of concrete fracture using interface elements. I: numerical model and tensile behavior. Materials and Structures. 2008;41:583-99.

[2] Yin A, Yang X, Gao H, Zhu H. Tensile fracture simulation of random heterogeneous asphalt mixture with cohesive crack model. Engineering Fracture Mechanics. 2012;92:40-55.

[3] Ren W, Yang Z, Sharma R, Zhang C, Withers PJ. Two-dimensional X-ray CT image based mesoscale fracture modelling of concrete. Engineering Fracture Mechanics. 2015;133:24-39.

[4] Alfaro MVC, Suiker ASJ, de Borst R. Transverse Failure Behavior of Fiber-epoxy Systems. Journal of Composite Materials. 2010;44:1493-516.

[5] Yang L, Yan Y, Liu Y, Ran Z. Microscopic failure mechanisms of fiber-reinforced polymer composites under transverse tension and compression. Composites Science and Technology. 2012;72:1818-25.

[6] Zhi J, Zhao L, Zhang J, Liu Z. A Numerical Method for Simulating the Microscopic Damage Evolution in Composites Under Uniaxial Transverse Tension. Applied Composite Materials. 2016;23:255-69.

[7] Sandino C, Kroliczek P, McErlain DD, Boyd SK. Predicting the permeability of trabecular bone by micro-computed tomography and finite element modeling. Journal of Biomechanics. 2014;47:3129-34.

[8] Chen Y, Pani M, Taddei F, Mazza C, Li X, Viceconti M. Large-Scale Finite Element Analysis of Human Cancellous Bone Tissue Micro Computer Tomography Data: A Convergence Study. Journal of Biomechanical Engineering-Transactions of the Asme. 2014;136:101013.

[9] Xu K, Xu X. Finite element analysis of mechanical properties of 3D five-directional braided 
composites. Materials Science and Engineering: A. 2008;487:499-509.

[10] Li D-s, Fang D-n, Jiang N, Xuefeng Y. Finite element modeling of mechanical properties of 3D five-directional rectangular braided composites. Composites Part B: Engineering. 2011;42:1373-85.

[11] Zhang C, Curiel-Sosa J, Bui TQ. Meso-scale progressive damage modeling and life prediction of 3D braided composites under fatigue tension loading. Composite Structures. 2018;201:62-71.

[12] Zhang C, Mao C, Curiel-Sosa JL, Bui TQ. Meso-Scale Finite Element Simulations of 3D Braided Textile Composites: Effects of Force Loading Modes. Applied Composite Materials. 2018;25:1-19.

[13] Xi X, Yang S, Li C-Q, Cai M, Hu X, Shipton ZK. Meso-scale mixed-mode fracture modelling of reinforced concrete structures subjected to non-uniform corrosion. Engineering Fracture Mechanics. 2018;199:114-30.

[14] Grassl P, Grégoire D, Solano LR, Pijaudier-Cabot G. Meso-scale modelling of the size effect on the fracture process zone of concrete. International Journal of Solids and Structures. 2012;49:1818-27.

[15] Benkemoun N, Hautefeuille M, Colliat JB, Ibrahimbegovic A. Failure of heterogeneous materials: 3D meso - scale FE models with embedded discontinuities. International Journal for Numerical Methods in Engineering. 2010;82:1671-88.

[16] Vaughan TJ, McCarthy CT. Micromechanical modelling of the transverse damage behaviour in fibre reinforced composites. Composites Science and Technology. 2011;71:388-96.

[17] Vaughan T, McCarthy C. A micromechanical study on the effect of intra-ply properties on transverse shear fracture in fibre reinforced composites. Composites Part A: Applied Science and Manufacturing. 2011;42:1217-28.

[18] Melro A, Camanho P, Pires FA, Pinho S. Micromechanical analysis of polymer composites reinforced by unidirectional fibres: Part I-Constitutive modelling. International Journal of Solids and Structures. 2013;50:1897-905.

[19] Barenblatt GI. The formation of equilibrium cracks during brittle fracture. General ideas and hypotheses. Axially-symmetric cracks. Journal of Applied Mathematics \& Mechanics. 1959;23:622-36.

[20] Dugdale DS. Yielding of steel sheets containing slits. Journal of the Mechanics and Physics of Solids. 1960;8:100-4.

[21] Needleman A. A continuum model for void nucleation by inclusion debonding. Journal of Applied Mechanics-Transactions of the Asme. 1987;54:525-31.

[22] Borst RD. Numerical aspects of cohesive-zone models. Engineering Fracture Mechanics. 2003;70:1743-57.

[23] Ortiz M, Pandolfi A. Finite-deformation irreversible cohesive elements for three-dimensional crackpropagation analysis. International Journal for Numerical Methods in Engineering. 1999;44:126782.

[24] Moes N, Belytschko T. Extended finite element method for cohesive crack growth. Engineering Fracture Mechanics. 2002;69:813-33.

[25] Hu X, Chen B, Tirvaudey M, Tan V, Tay T. Integrated XFEM-CE analysis of delamination migration in multi-directional composite laminates. Composites Part A: Applied Science and Manufacturing. 2016;90:161-73.

[26] Fang X, Yang Q, Cox B, Zhou Z. An augmented cohesive zone element for arbitrary crack coalescence and bifurcation in heterogeneous materials. International Journal for Numerical Methods in Engineering. 2011;88:841-61.

[27] Lu X, Chen B, Tan V, Tay T. A separable cohesive element for modelling coupled failure in laminated composite materials. Composites Part A: Applied Science and Manufacturing. 2018;107:387-98.

[28] Griffith AA. The Phenomena of Rupture and Flow in Solids. Philosophical Transactions of the Royal Society of London. 1921;221:163-98.

[29] Francfort GA, Marigo JJ. Revisiting brittle fracture as an energy minimization problem. Journal of the Mechanics \& Physics of Solids. 1998;46:1319-42.

[30] Ambrosio L, Tortorelli VM. On the approximation of free discontinuity problems. Bollettino Della Unione Matematica Italiana. 1992;6B:105-23.

[31] Bourdin B, Chambolle A. Implementation of an adaptive finite-element approximation of the Mumford-Shah functional. Numerische Mathematik. 2000;85:609-46.

[32] Fraternali F. Free discontinuity finite element models in two-dimensions for in-plane crack problems. Theoretical \& Applied Fracture Mechanics. 2007;47:274-82.

[33] Schmidt B, Fraternali F, Ortiz M. Eigenfracture: an eigendeformation approach to variational fracture. Multiscale Modeling \& Simulation. 2009;7:1237-66.

[34] Wu J-Y, Nguyen VP, Nguyen CT, Sutula D, Bordas S, Sinaie S. Phase field modeling of fracture. 
Advances in Applied Mechancis: Multi-scale Theory and Computation. 2018;52.

[35] Chambolle A, Francfort GA, Marigo JJ. When and how do cracks propagate? Journal of the Mechanics \& Physics of Solids. 2009;57:1614-22.

[36] Gerasimov T, Noii N, Allix O, De Lorenzis L. A non-intrusive global/local approach applied to phase-field modeling of brittle fracture. Advanced Modeling and Simulation in Engineering Sciences. 2018;5:14.

[37] Patil RU, Mishra BK, Singh IV. An adaptive multiscale phase field method for brittle fracture. Computer Methods in Applied Mechanics \& Engineering. 2017;329:254-88.

[38] Molnár G, Gravouil A. 2D and 3D Abaqus implementation of a robust staggered phase-field solution for modeling brittle fracture. Finite Elements in Analysis \& Design. 2017;130:27-38.

[39] Mesgarnejad A, Bourdin B, Khonsari M. Validation simulations for the variational approach to fracture. Computer Methods in Applied Mechanics and Engineering. 2015;290:420-37.

[40] Miehe C, Welschinger F, Hofacker M. Thermodynamically consistent phase-field models of fracture: Variational principles and multi-field FE implementations. International Journal for Numerical Methods in Engineering. 2010;83:1273-311.

[41] Miehe C, Hofacker M, Welschinger F. A phase field model for rate-independent crack propagation: Robust algorithmic implementation based on operator splits. Computer Methods in Applied Mechanics \& Engineering. 2010;199:2765-78.

[42] Ambati M, Gerasimov T, Lorenzis LD. A review on phase-field models of brittle fracture and a new fast hybrid formulation. Computational Mechanics. 2015;55:383-405.

[43] Tanne E, Li T, Bourdin B, Marigo JJ, Maurini C. Crack nucleation in variational phase-field models of brittle fracture. Journal of the Mechanics and Physics of Solids. 2018;110:80-99.

[44] Bourdin B, Larsen CJ, Richardson CL. A time-discrete model for dynamic fracture based on crack regularization. International Journal of Fracture. 2011;168:133-43.

[45] Borden MJ, Verhoosel CV, Scott MA, Hughes TJR, Landis CM. A phase-field description of dynamic brittle fracture. Computer Methods in Applied Mechanics and Engineering. 2012;217:7795.

[46] Doan DH, Bui TQ, Duc ND, Fushinobu K. Hybrid phase field simulation of dynamic crack propagation in functionally graded glass-filled epoxy. Composites Part B Engineering. 2016;99:266-76.

[47] Doan DH, Bui TQ, Van Do T, Duc ND. A rate-dependent hybrid phase field model for dynamic crack propagation. Journal of Applied Physics. 2017;122:115102.

[48] Larsen CJ, Ortner C, Süli E. Existence of solutions to a regularized model of dynamic fracture. Mathematical Models and Methods in Applied Sciences. 2010;20:1021-48.

[49] Roy P, Deepu S, Pathrikar A, Roy D, Reddy J. Phase field based peridynamics damage model for delamination of composite structures. Composite Structures. 2017;180:972-93.

[50] Li B, Peco C, Millán D, Arias I, Arroyo M. Phase - field modeling and simulation of fracture in brittle materials with strongly anisotropic surface energy. International Journal for Numerical Methods in Engineering. 2015;102:711-27.

[51] Teichtmeister S, Kienle D, Aldakheel F, Keip MA. Phase field modeling of fracture in anisotropic brittle solids. International Journal of Non-Linear Mechanics. 2017;97:1-21.

[52] Verhoosel CV, De Borst R, Eacute. A phase - field model for cohesive fracture. International Journal for Numerical Methods in Engineering. 2013;96:43-62.

[53] Freddi F, Iurlano F. Numerical insight of a variational smeared approach to cohesive fracture. Journal of the Mechanics \& Physics of Solids. 2017;98:156-71.

[54] Wu JY. A unified phase-field theory for the mechanics of damage and quasi-brittle failure. Journal of the Mechanics \& Physics of Solids. 2017;103:72-99.

[55] Nguyen VP, Wu J-Y. Modeling dynamic fracture of solids with a phase-field regularized cohesive zone model. Computer Methods in Applied Mechanics and Engineering. 2018;340:1000-22.

[56] Paggi M, Reinoso J. Revisiting the problem of a crack impinging on an interface: a modeling framework for the interaction between the phase field approach for brittle fracture and the interface cohesive zone model. Computer Methods in Applied Mechanics and Engineering. 2017;321:14572 .

[57] Carollo V, Reinoso J, Paggi M. Modeling complex crack paths in ceramic laminates: A novel variational framework combining the phase field method of fracture and the cohesive zone model. Journal of the European Ceramic Society. 2018;38:2994-3003.

[58] Carollo V, Reinoso J, Paggi M. A 3D finite strain model for intralayer and interlayer crack simulation coupling the phase field approach and cohesive zone model. Composite Structures. 2017;182:636-51. 
[59] Paggi M, Corrado M, Reinoso J. Fracture of solar-grade anisotropic polycrystalline Silicon: A combined phase field-cohesive zone model approach. Computer Methods in Applied Mechanics and Engineering. 2018;330:123-48.

[60] Nguyen T-T, Yvonnet J, Zhu Q-Z, Bornert M, Chateau C. A phase-field method for computational modeling of interfacial damage interacting with crack propagation in realistic microstructures obtained by microtomography. Computer Methods in Applied Mechanics and Engineering. 2016;312:567-95.

[61] Xia L, Yvonnet J, Ghabezloo S. Phase field modeling of hydraulic fracturing with interfacial damage in highly heterogeneous fluid-saturated porous media. Engineering Fracture Mechanics. 2017;186:158-80.

[62] Xia L, Da D, Yvonnet J. Topology optimization for maximizing the fracture resistance of quasibrittle composites. Computer Methods in Applied Mechanics and Engineering. 2018;332:234-54.

[63] Msekh MA, Cuong N, Zi G, Areias P, Zhuang X, Rabczuk T. Fracture properties prediction of clay/epoxy nanocomposites with interphase zones using a phase field model. Engineering Fracture Mechanics. 2018;188:287-99.

[64] Msekh MA, Silani M, Jamshidian M, Areias P, Zhuang X, Zi G, et al. Predictions of J integral and tensile strength of clay/epoxy nanocomposites material using phase field model. Composites Part B: Engineering. 2016;93:97-114.

[65] Hansen-Dörr AC, de Borst R, Hennig P, Kästner M. Phase-field modelling of interface failure in brittle materials. Computer Methods in Applied Mechanics and Engineering. 2018.

[66] Simulia DCS. Abaqus 6.11 analysis user's manual. 2011.

[67] Bourdin B, Francfort GA, Marigo JJ. The Variational Approach to Fracture: Springer Science+Business Media B.V; 2008.

[68] Amor H, Marigo J-J, Maurini C. Regularized formulation of the variational brittle fracture with unilateral contact: Numerical experiments. Journal of the Mechanics and Physics of Solids. 2009;57:1209-29.

[69] Wu JY. A geometrically regularized gradient-damage model with energetic equivalence. Computer Methods in Applied Mechanics \& Engineering. 2017;328:612-37.

[70] Zhang P, Hu X, Yao W. An iteration scheme for phase field model for cohesive fracture and its implementation in Abaqus. Engineering Fracture Mechanics. 2018;204:268-87.

[71] Bourdin B, Marigo J-J, Maurini C, Sicsic P. Morphogenesis and propagation of complex cracks induced by thermal shocks. Physical review letters. 2014;112:014301.

[72] Borden MJ, Verhoosel CV, Scott MA, Hughes TJ, Landis CM. A phase-field description of dynamic brittle fracture. Computer Methods in Applied Mechanics and Engineering. 2012;217:77-95.

[73] Karihaloo BL, Xiao QZ. Modelling of stationary and growing cracks in FE framework without remeshing: a state-of-the-art review. Computers \& Structures. 2003;81:119-29.

[74] Nguyen VP. Discontinuous Galerkin/extrinsic cohesive zone modeling: Implementation caveats and applications in computational fracture mechanics. Engineering fracture mechanics. 2014;128:3768.

[75] Labanda NA, Giusti SM, Luccioni BM. Meso-scale fracture simulation using an augmented Lagrangian approach. International Journal of Damage Mechanics. 2018;27:138-75.

[76] Nguyen VP, Nguyen GD, Nguyen CT, Shen L, Dias-da-Costa D, El-Zein A, et al. Modelling complex cracks with finite elements: a kinematically enriched constitutive model. International Journal of Fracture. 2017;203:21-39.

[77] Paris F, Correa E, Mantic V. Kinking of transversal interface cracks between fiber and matrix. Journal of Applied Mechanics-Transactions of the Asme. 2007;74:703-16.

\section{Figure Captions}

Figure 1. Sharp (a) and diffusive (b) representation of interface and crack.

Figure 2. (a) Sharp interface in a 1-D bar. (b) Exponential interface phase field.

Figure 3. Material properties (a) for sharp and (b) diffusive interfaces.

Figure 4. Complex failure mechanisms of a body containing an inclusion. (a) Matrix cracking and interface debonding. (b) Interaction between matrix cracking and interface debonding.

Figure 5. (a) Sharp and (b) diffusive crack passing through the smeared interface. 
Figure 6. A notched beam subjected to four-point bending.

Figure 7. Deformation and the crack pattern (represented by phase field) at $\bar{u}=-1.2 \mathrm{~mm}$ for

$$
l_{0} / l_{i}=1.0 .
$$

Figure 8. Numerical results of the effective critical energy release rate with different length scale ratios.

Figure 9. Pixelated image of the sample with inclusion and voids.

Figure 10. Distinguish different elements and interface nodes from a regular FE mesh.

Figure 11. Single fiber system under transverse tension.

Figure 12. Crack evolution in the single fiber system according to [77].

Figure 13. Fiber elements and interface nodes provided by image process method.

Figure 14. Smeared interface represented by the interface phase field for $l_{i}=0.025 \mathrm{~mm}$.

Figure 15. Crack patterns represented by crack phase field for $l_{0} / l_{i}=1.0$ at (a) $u=0.0075 \mathrm{~mm}$

$$
\text { and (b) } . u=0.03 \mathrm{~mm}
$$

Figure 16. Crack pattern obtained by Nguyen et al. [76]. (Reprinted with permission from

$$
\text { Springer) }
$$

Figure 17. Load-displacement curves for the four different internal

$$
\text { length scale ratios. }
$$

Figure 18. Crack patterns represented by crack phase field for (a) $l_{0}=0.025 \mathrm{~mm}$, (b)

$$
l_{0}=0.035 \mathrm{~mm}, \text { (c) } l_{0}=0.05 \mathrm{~mm} .
$$

Figure 19. Load-displacement curves for different $l_{0}$.

Figure 20. Load-displacement curves with sufficiently small $l_{0}$.

Figure 21. Meso-scale structure of concrete under tension [3]. (Reprinted with permission from

Elsevier)

Figure 22. Recognized (a) inclusion elements, interface nodes and (b) void elements.

Figure 23. Smeared interface represented by the interface phase field for $l_{i}=0.2325 \mathrm{~mm}$.

Figure 24. Stress-displacement curves with different $l_{0}$.

Figure 25. Crack patterns at different stages in Figure 24.

Figure 26. Total iteration steps and computational time for different $l_{0}$.

Figure 27. Iteration steps at each loading increment for different $l_{0}$. 


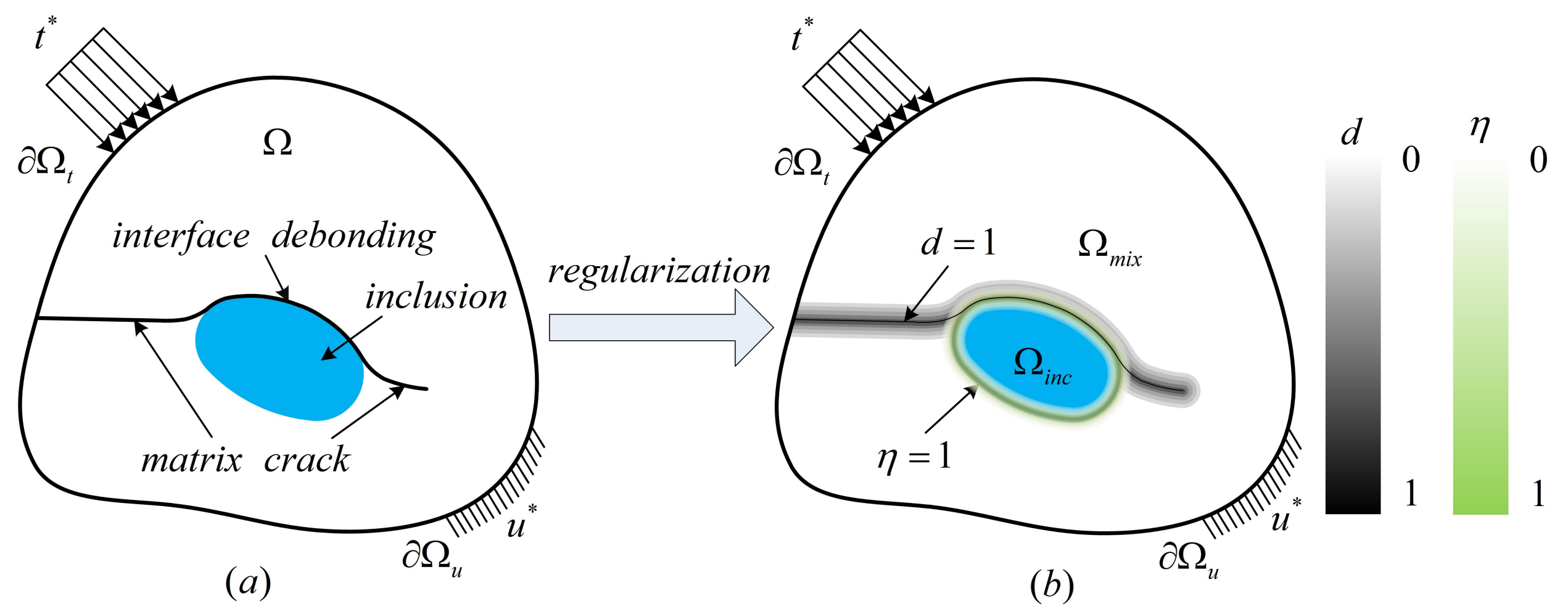




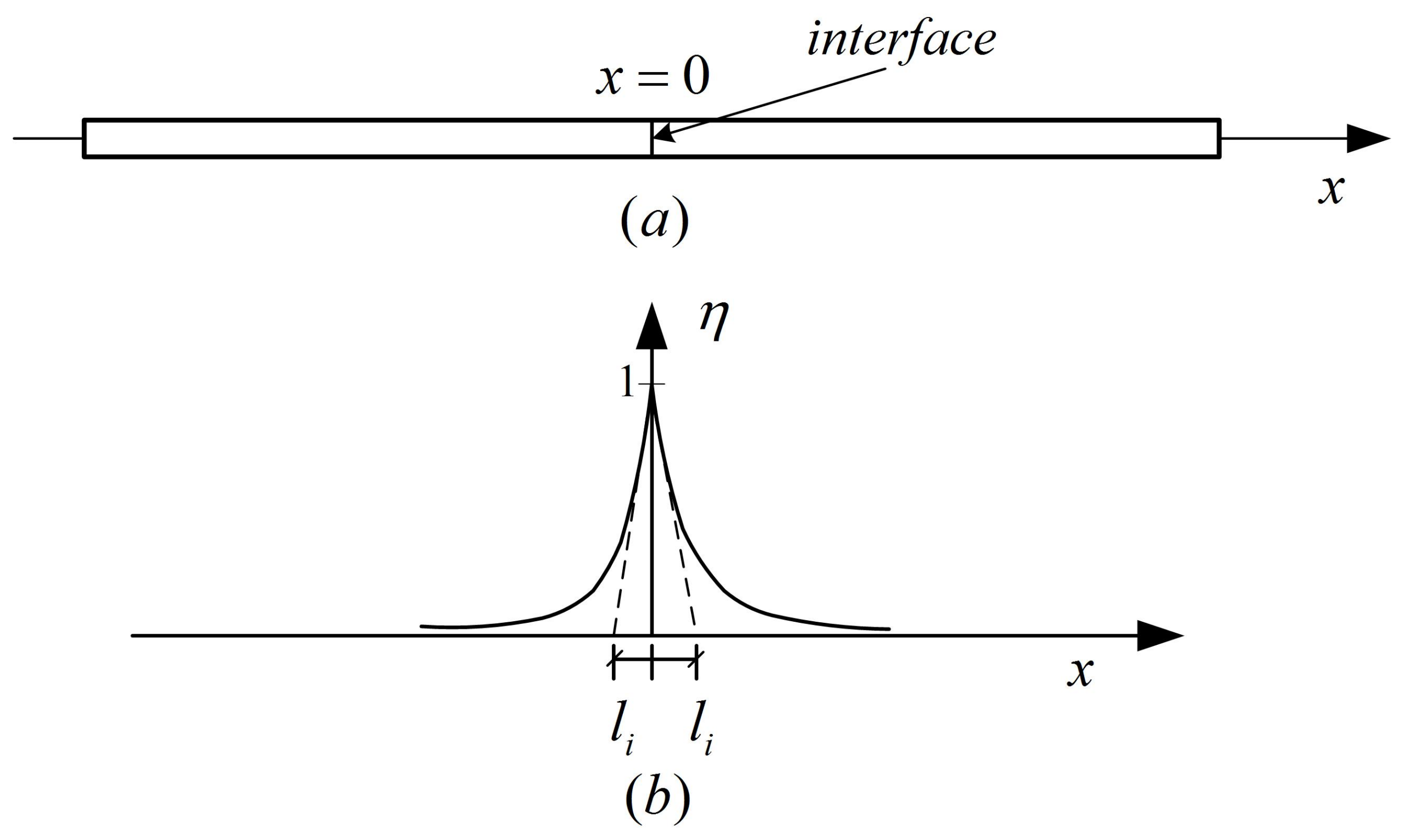




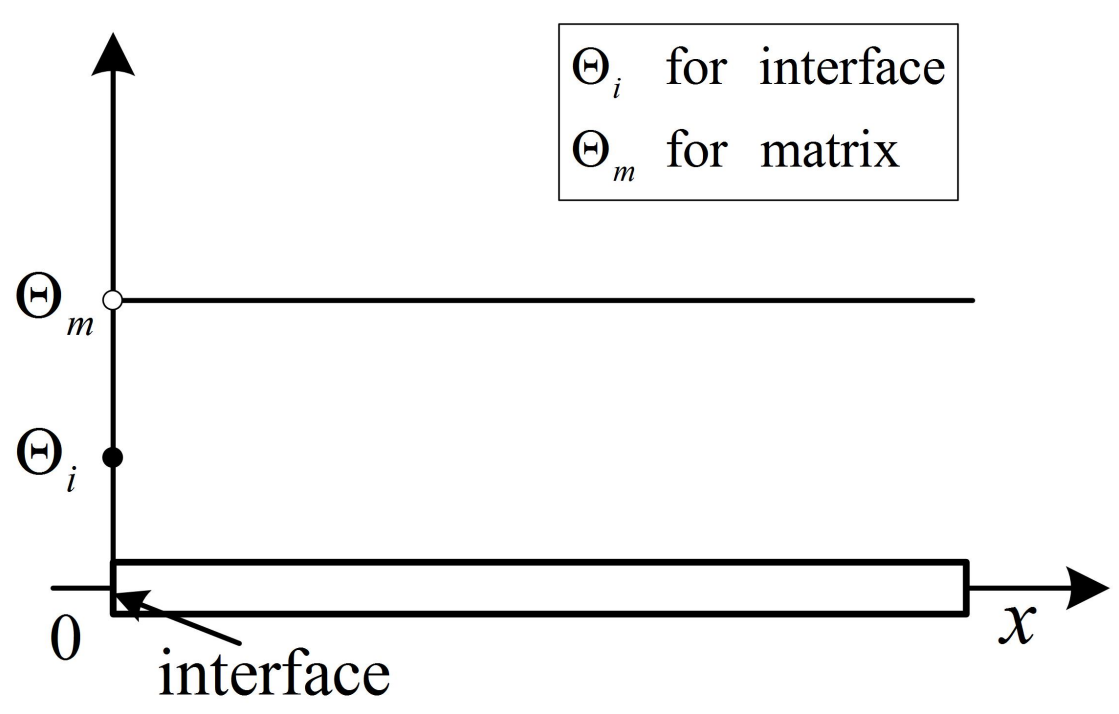

(a)

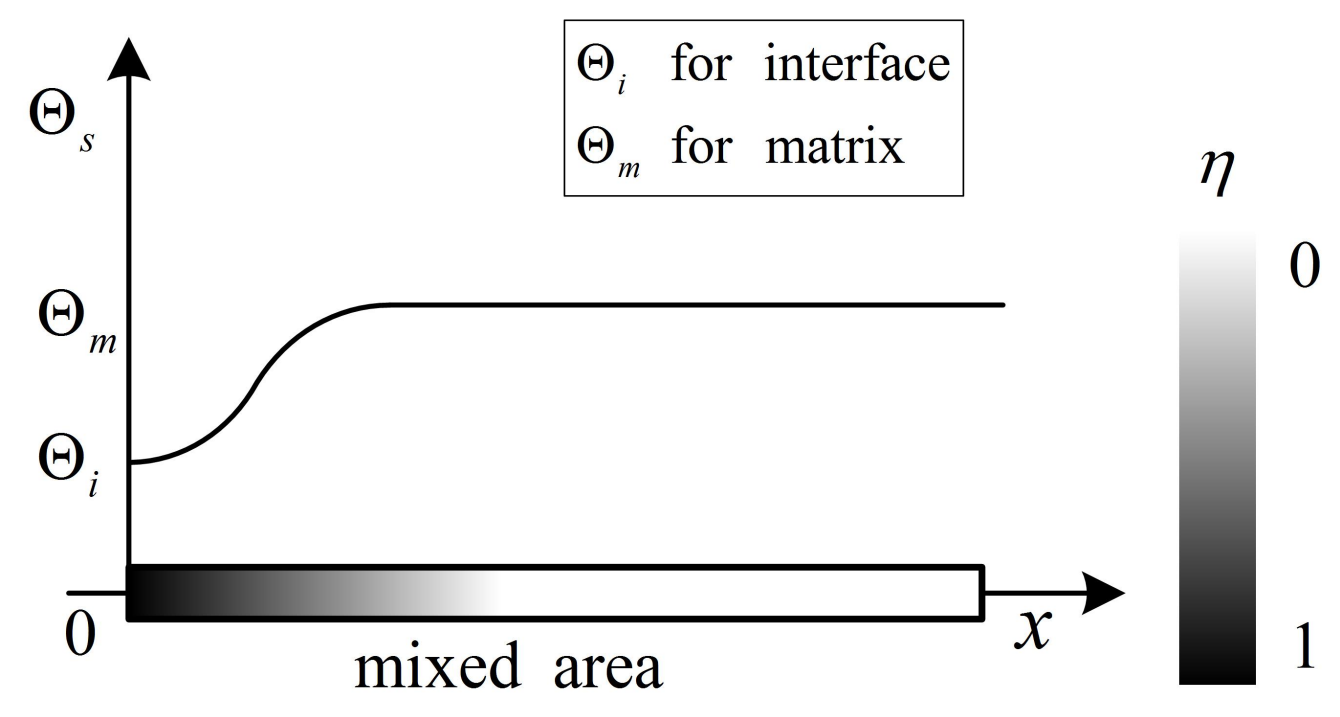

(b) 


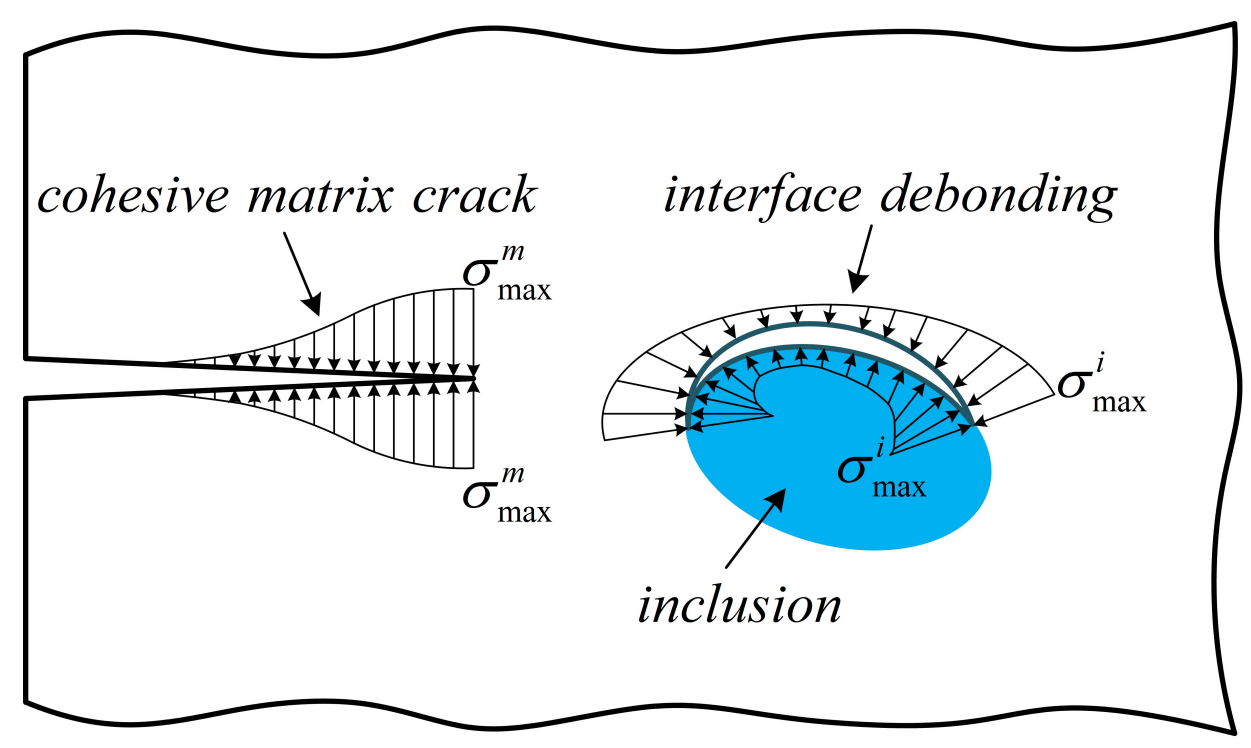

(a)

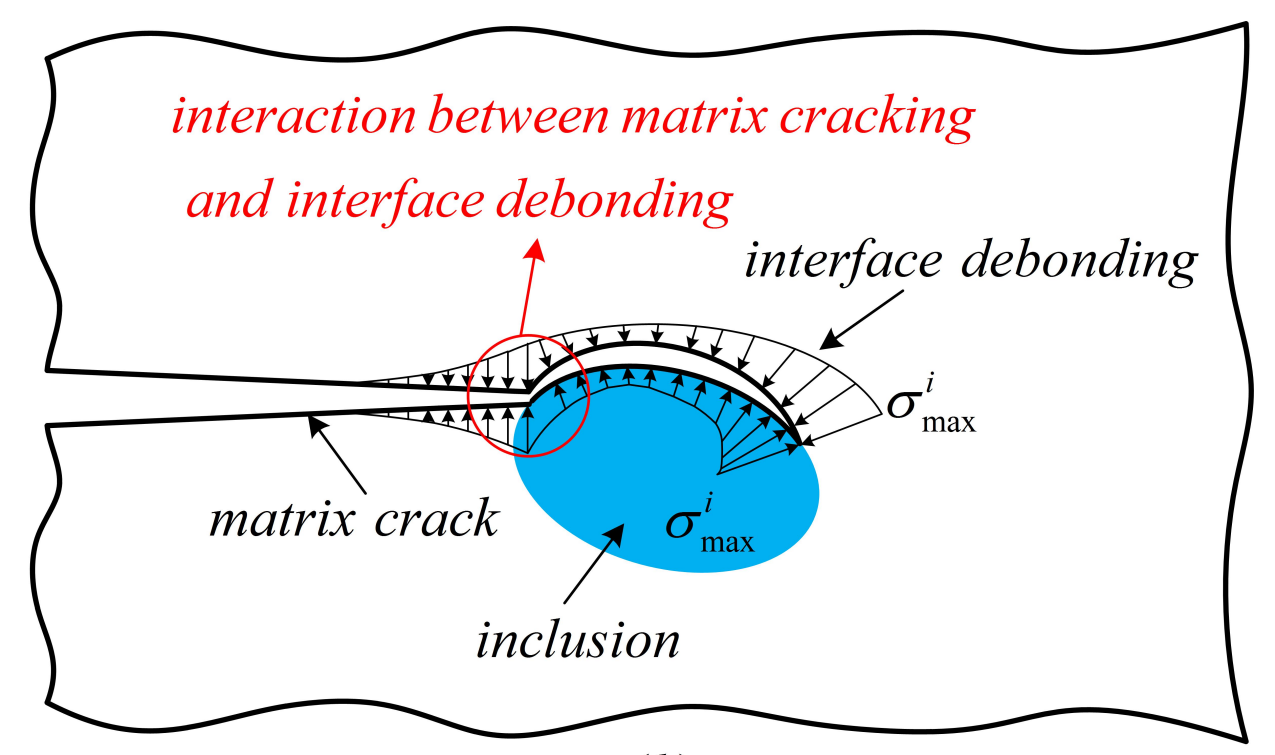

(b) 


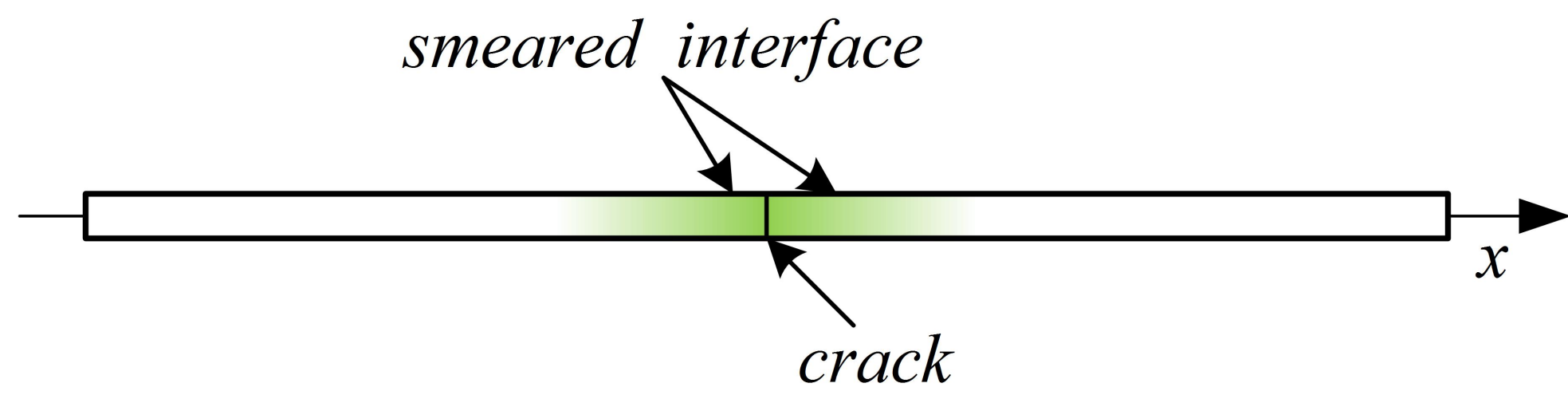

(b)

$\eta$

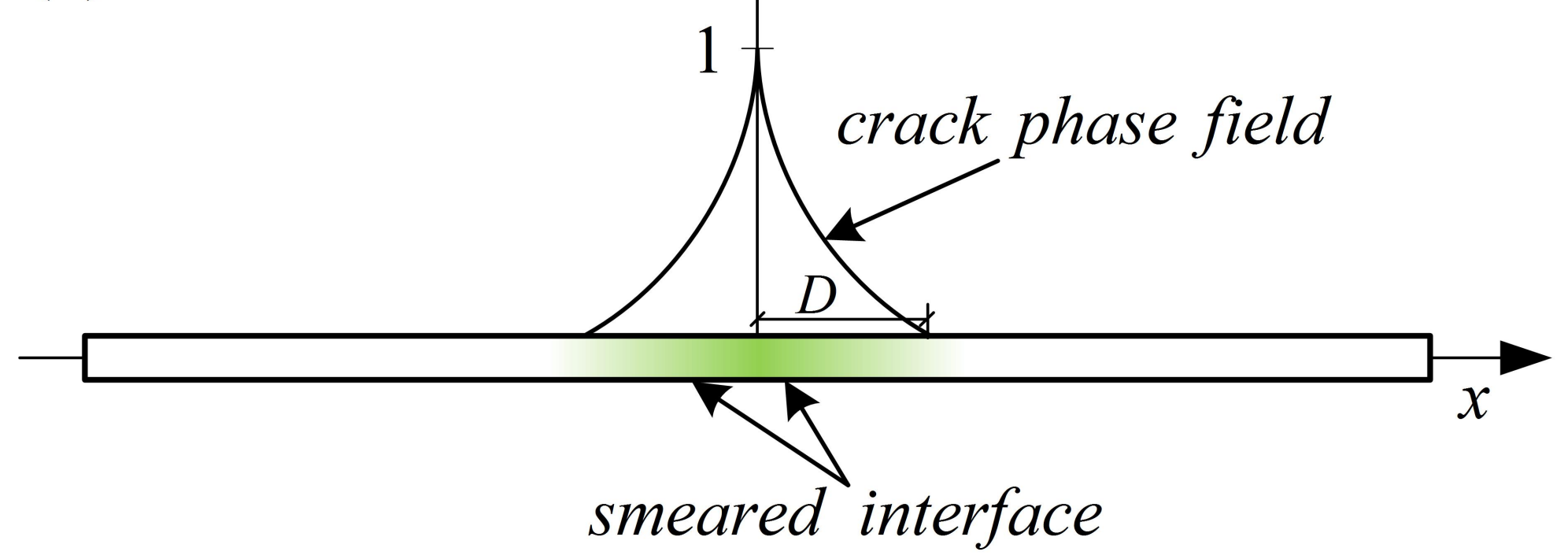

0 


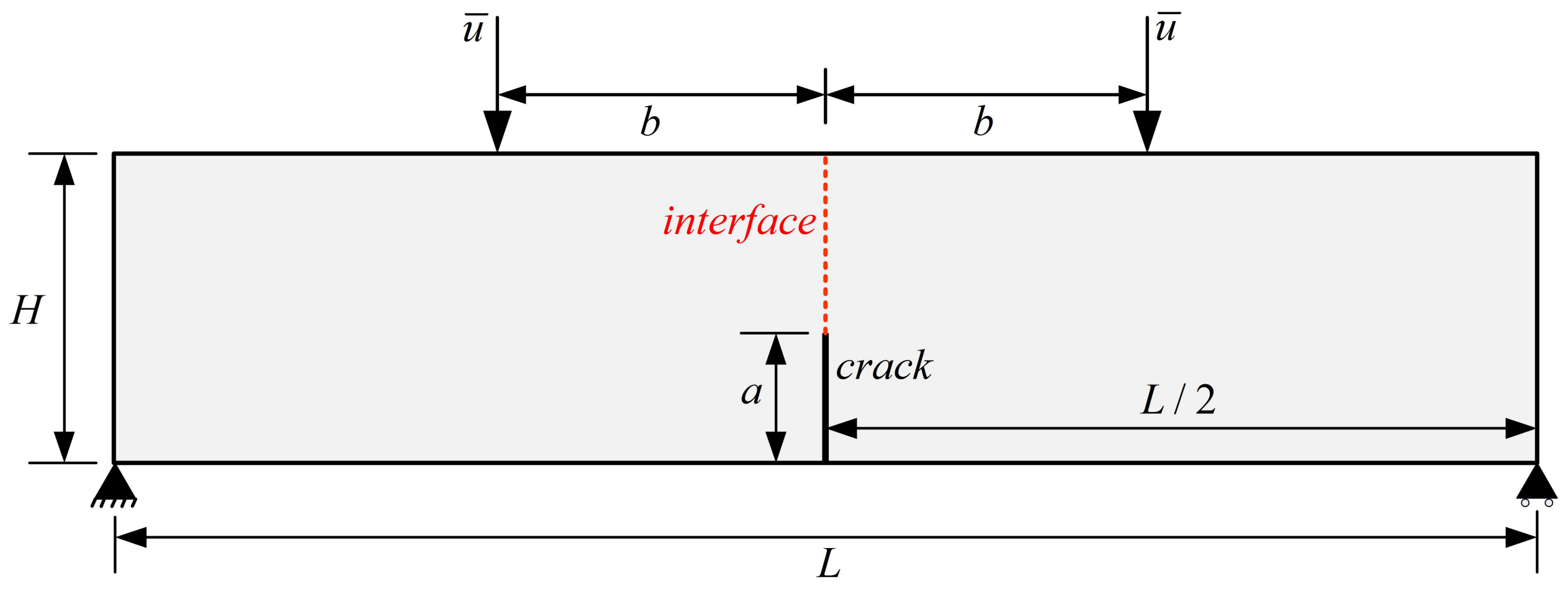




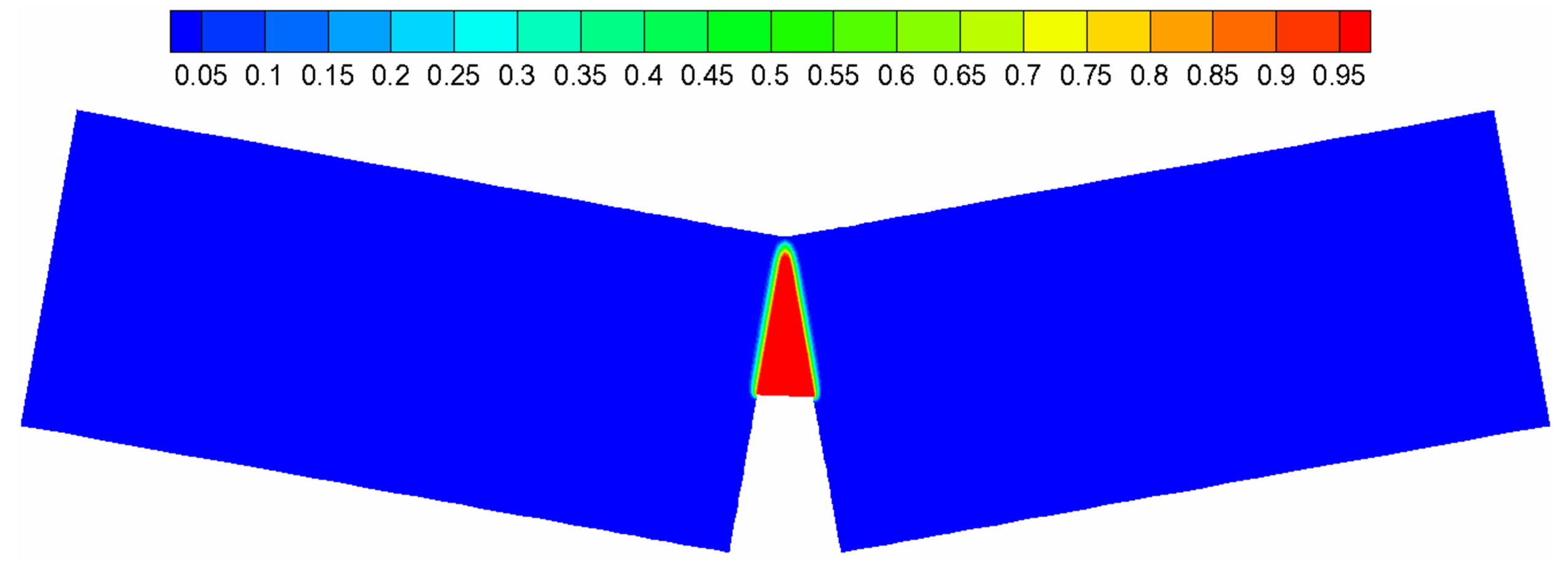




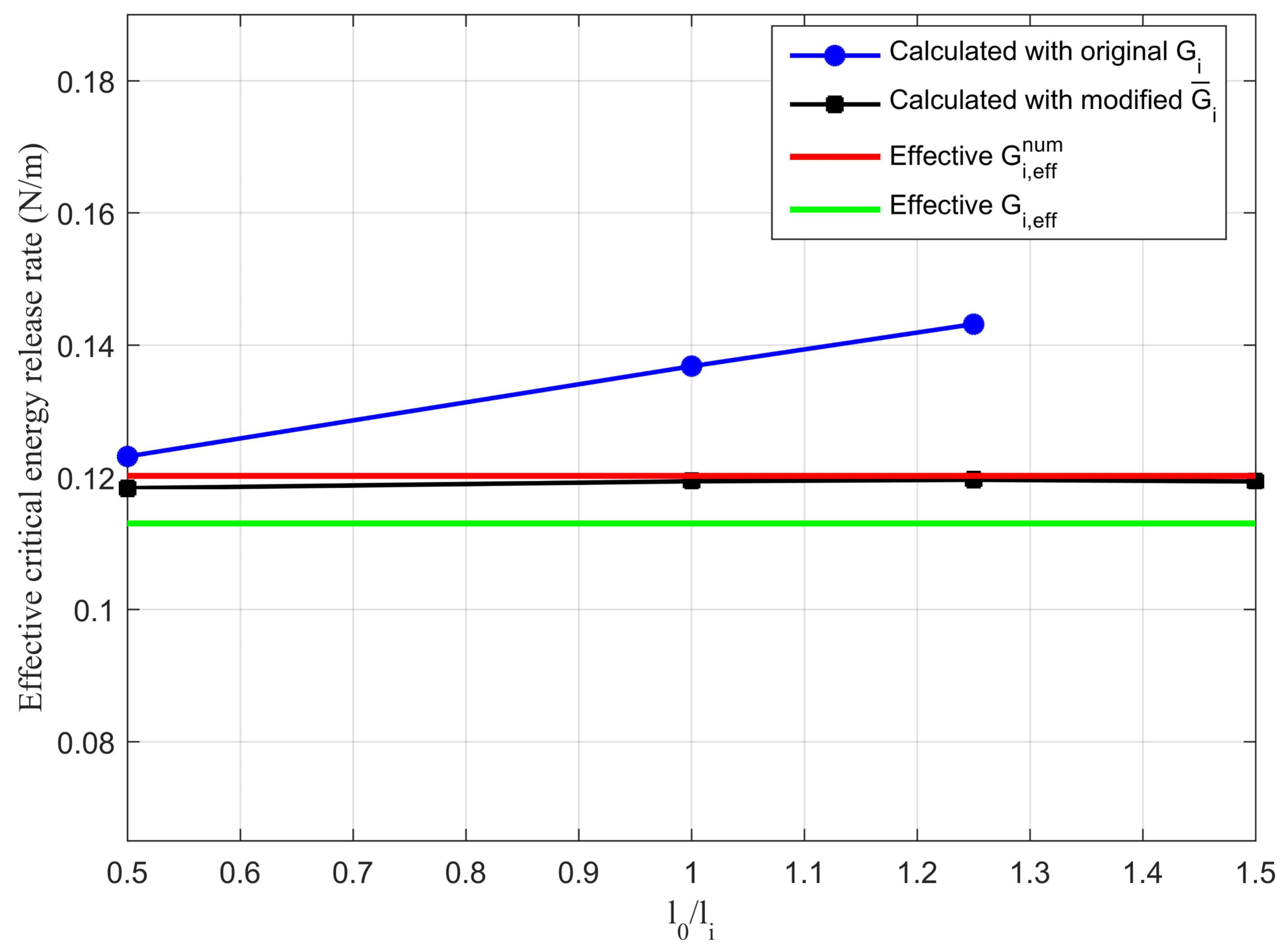




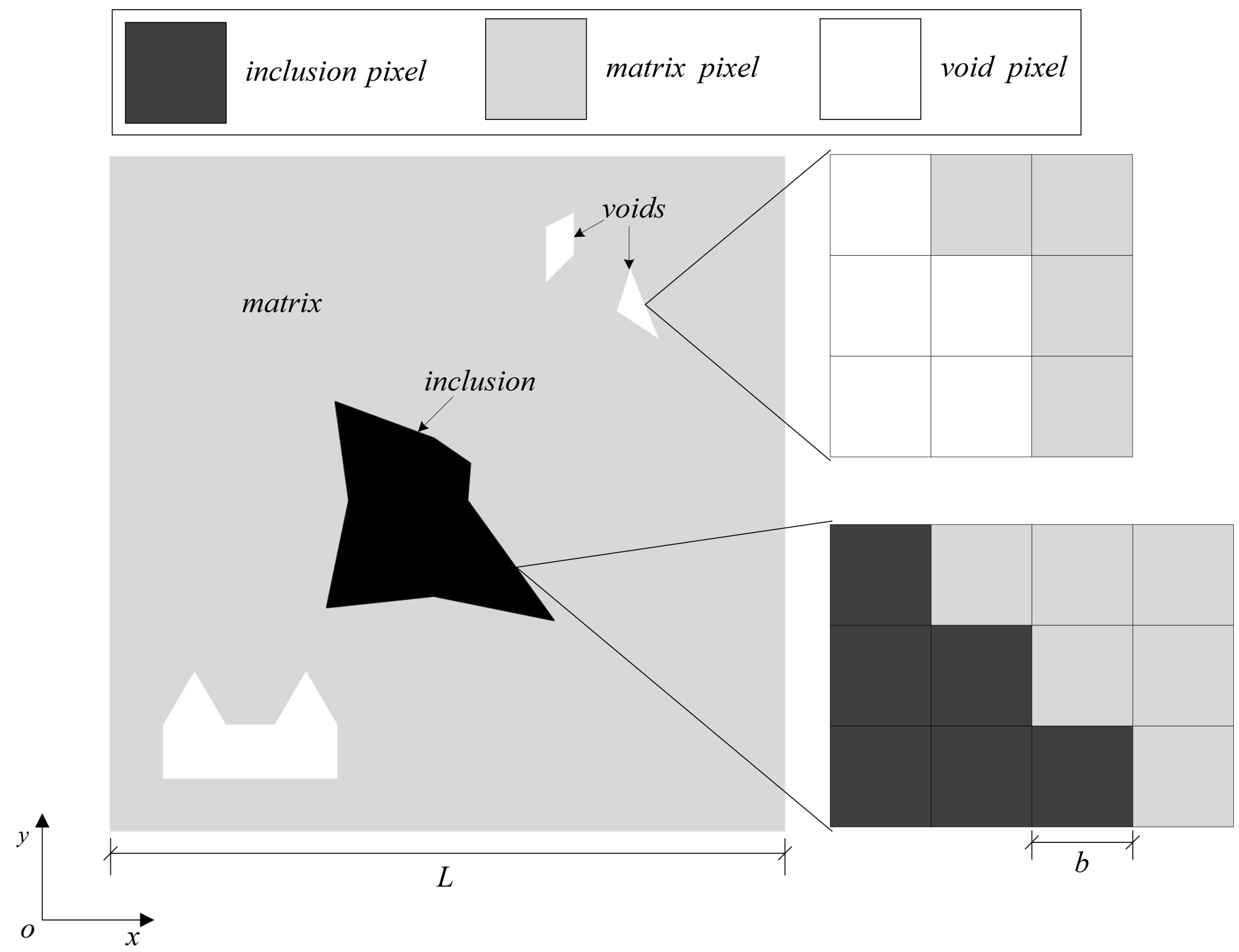




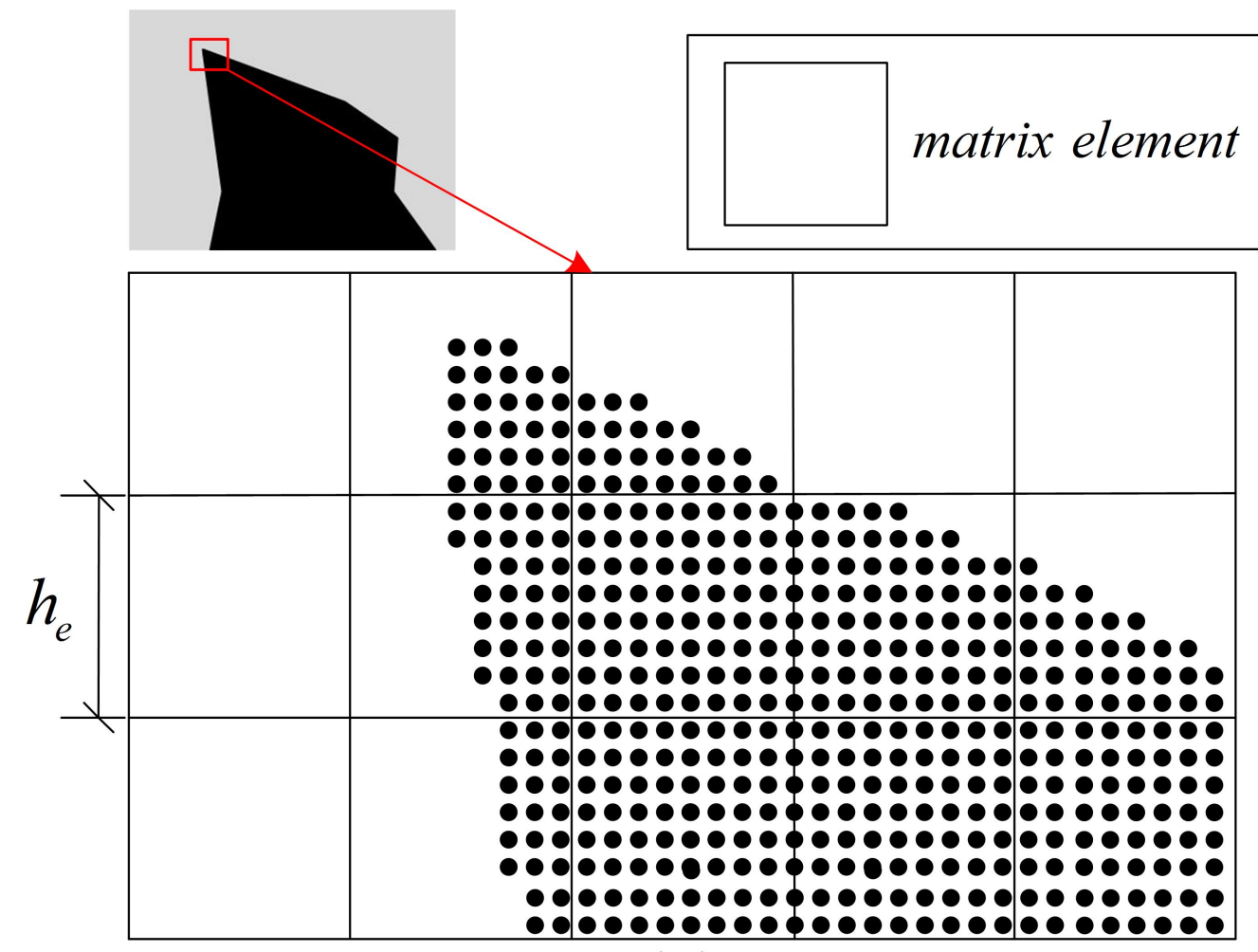

(a)

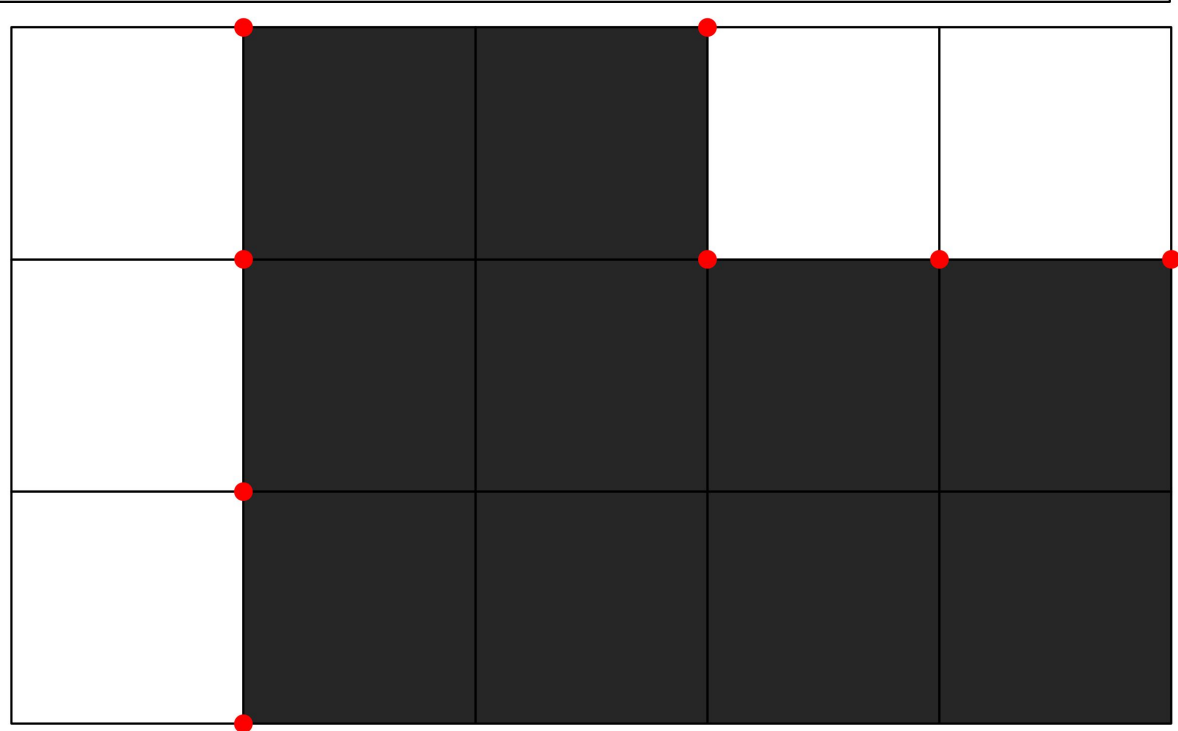

(b) 


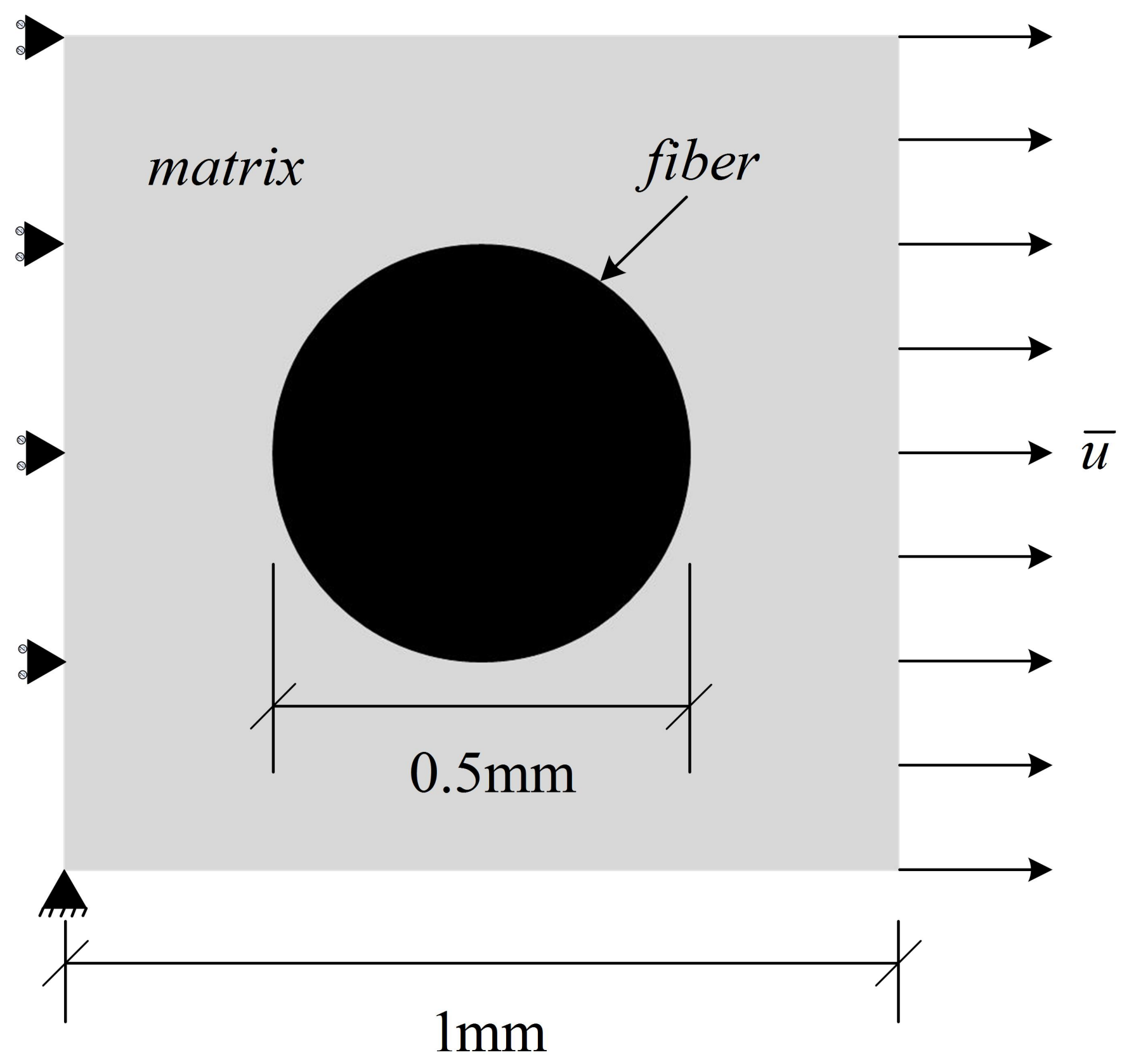




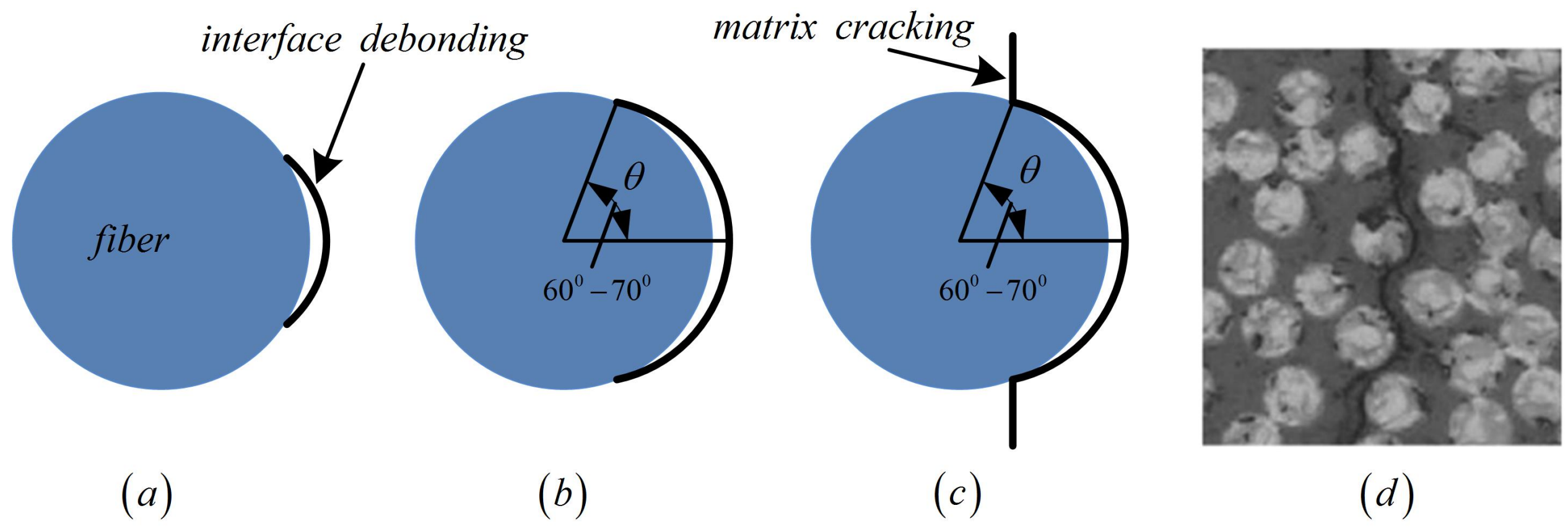




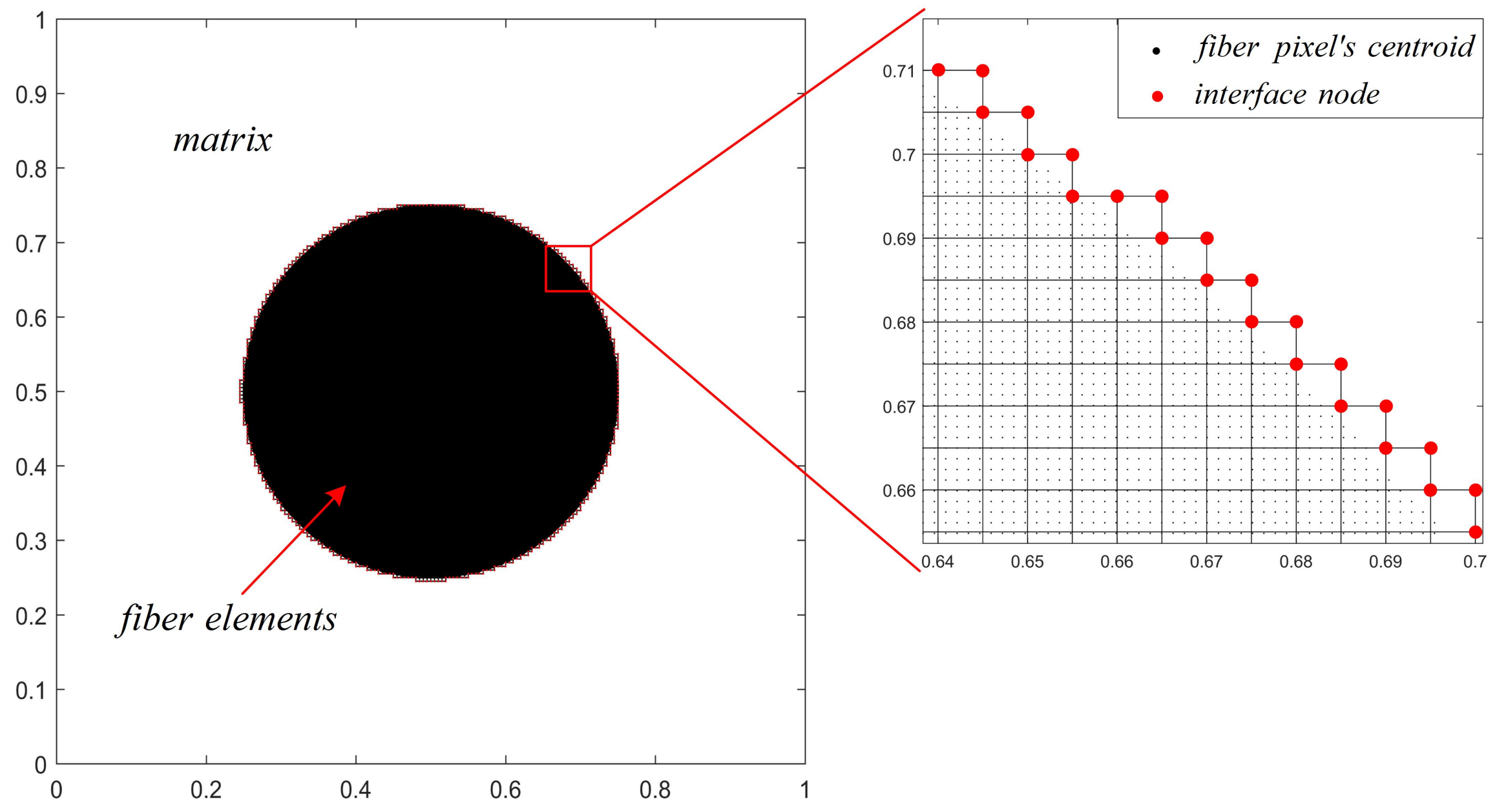




\begin{tabular}{|l|l|}
\hline & 0.95 \\
\hline 0.9 \\
\hline 0.85 \\
0.8 \\
\hline 0.75 \\
0.7 \\
\hline 0.65 \\
\hline 0.6 \\
\hline 0.55 \\
\hline 0.5 \\
\hline 0.45 \\
0.4 \\
\hline 0.35 \\
0.3 \\
0.25 \\
\hline 0.2 \\
0.15 \\
0.1 \\
\hline 0.05 \\
\hline
\end{tabular}



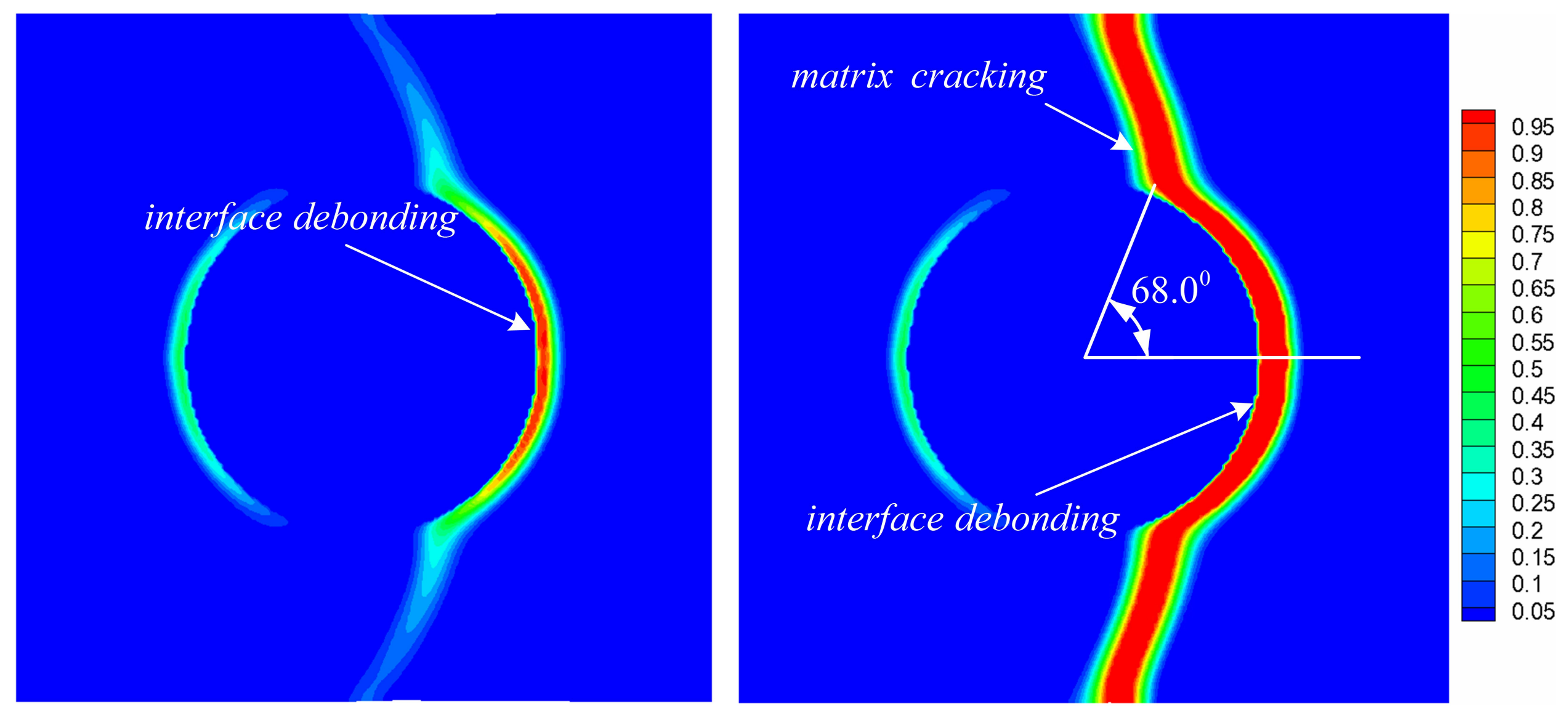

(a)

(b) 


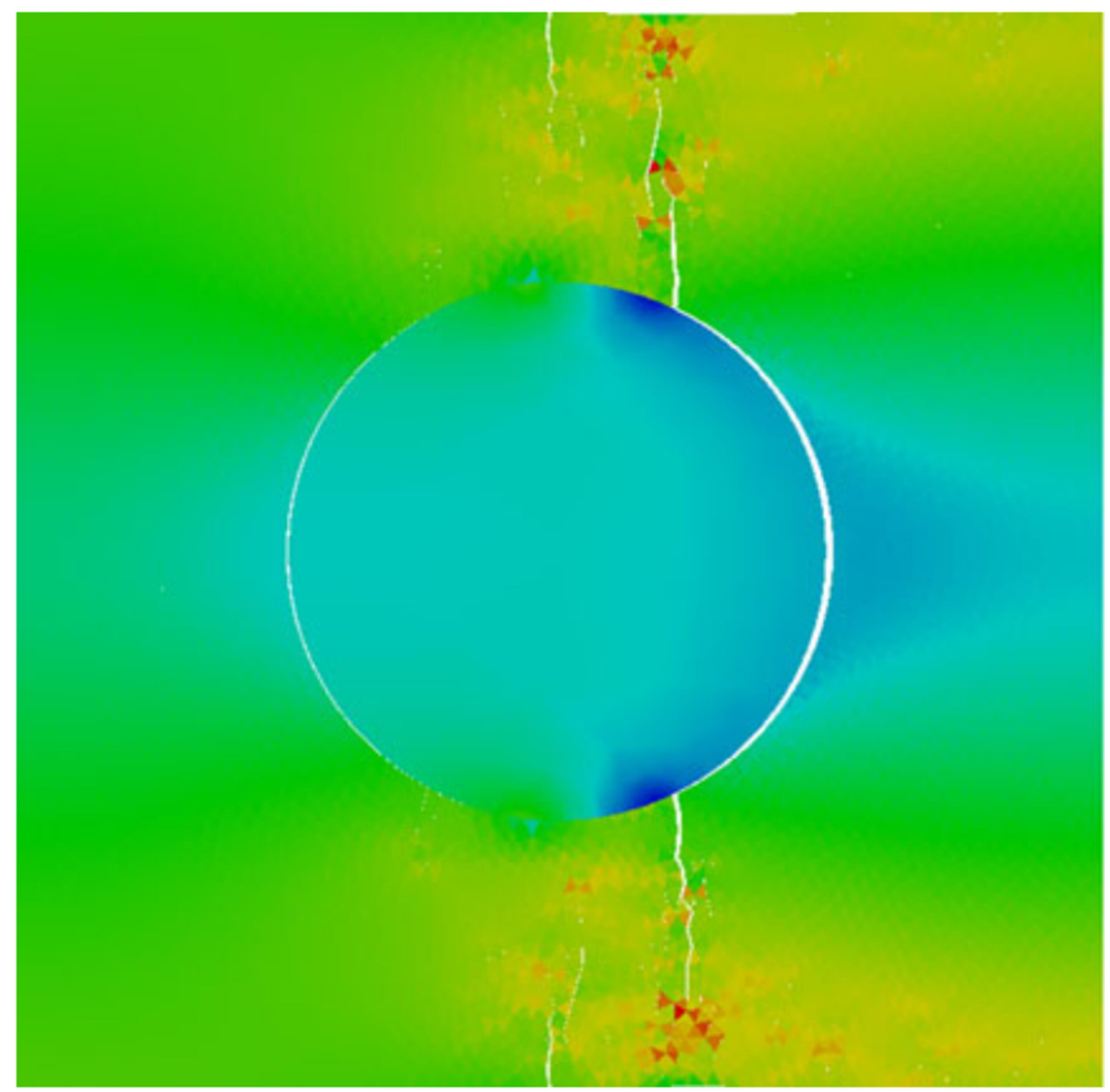




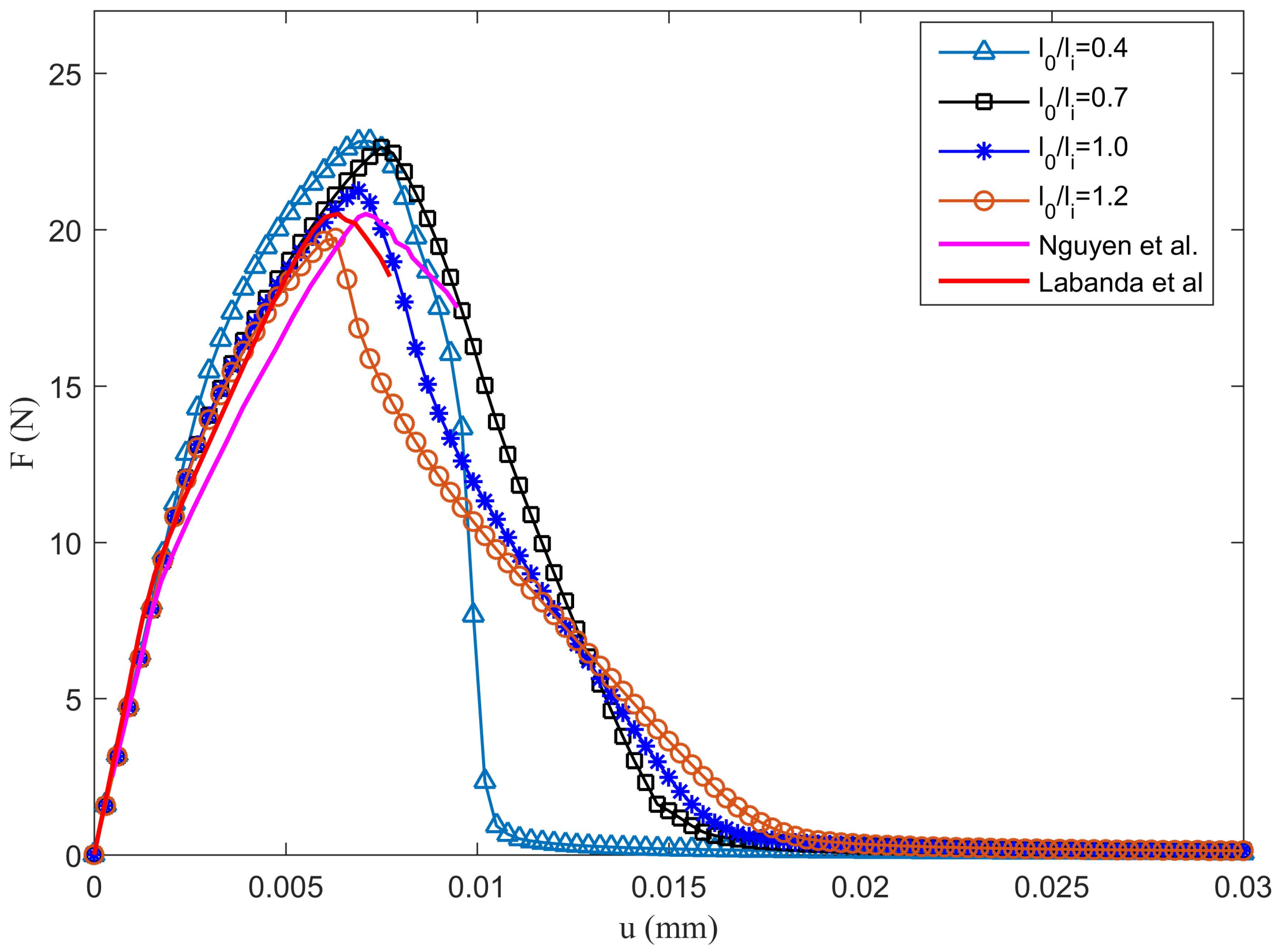




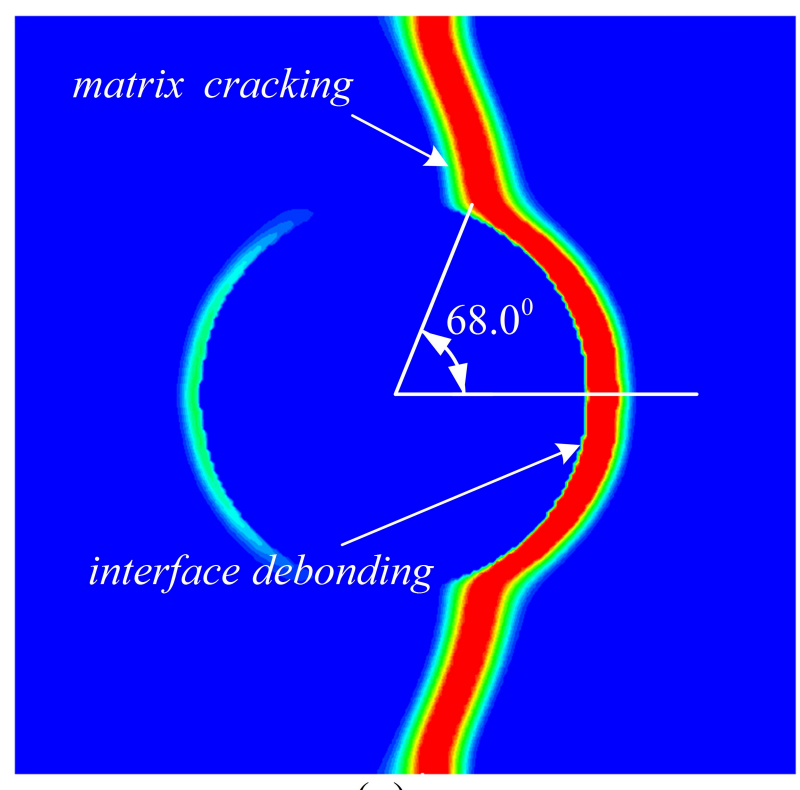

(a)

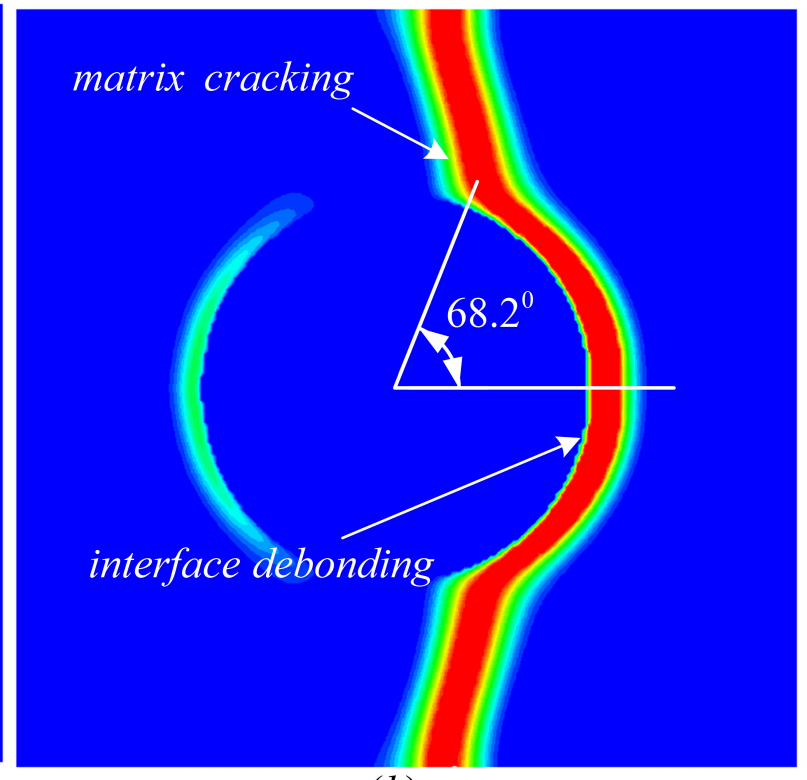

(b)

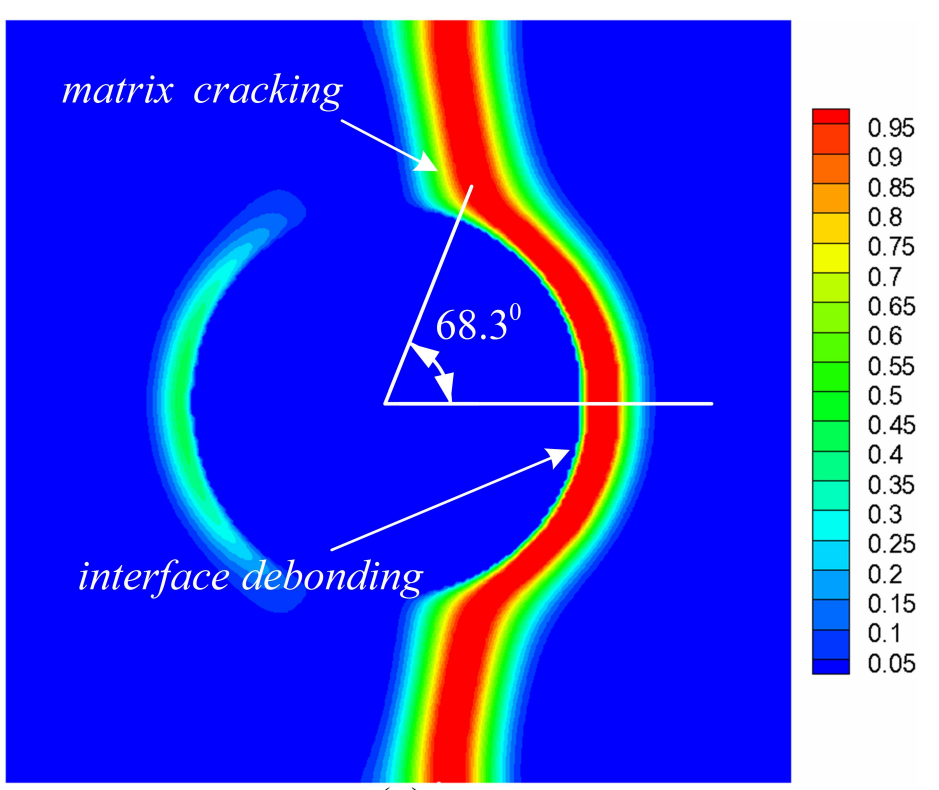

(c) 


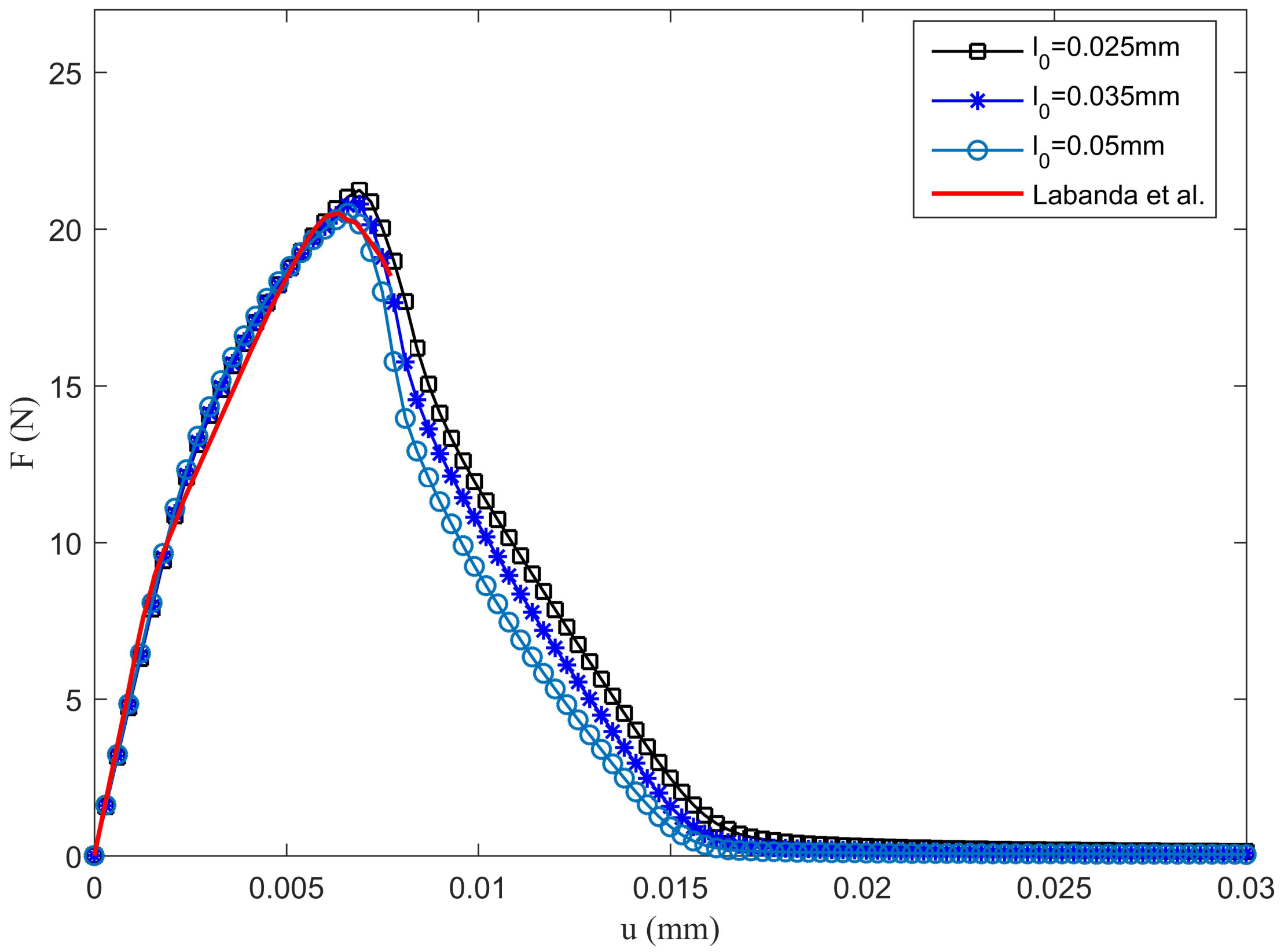




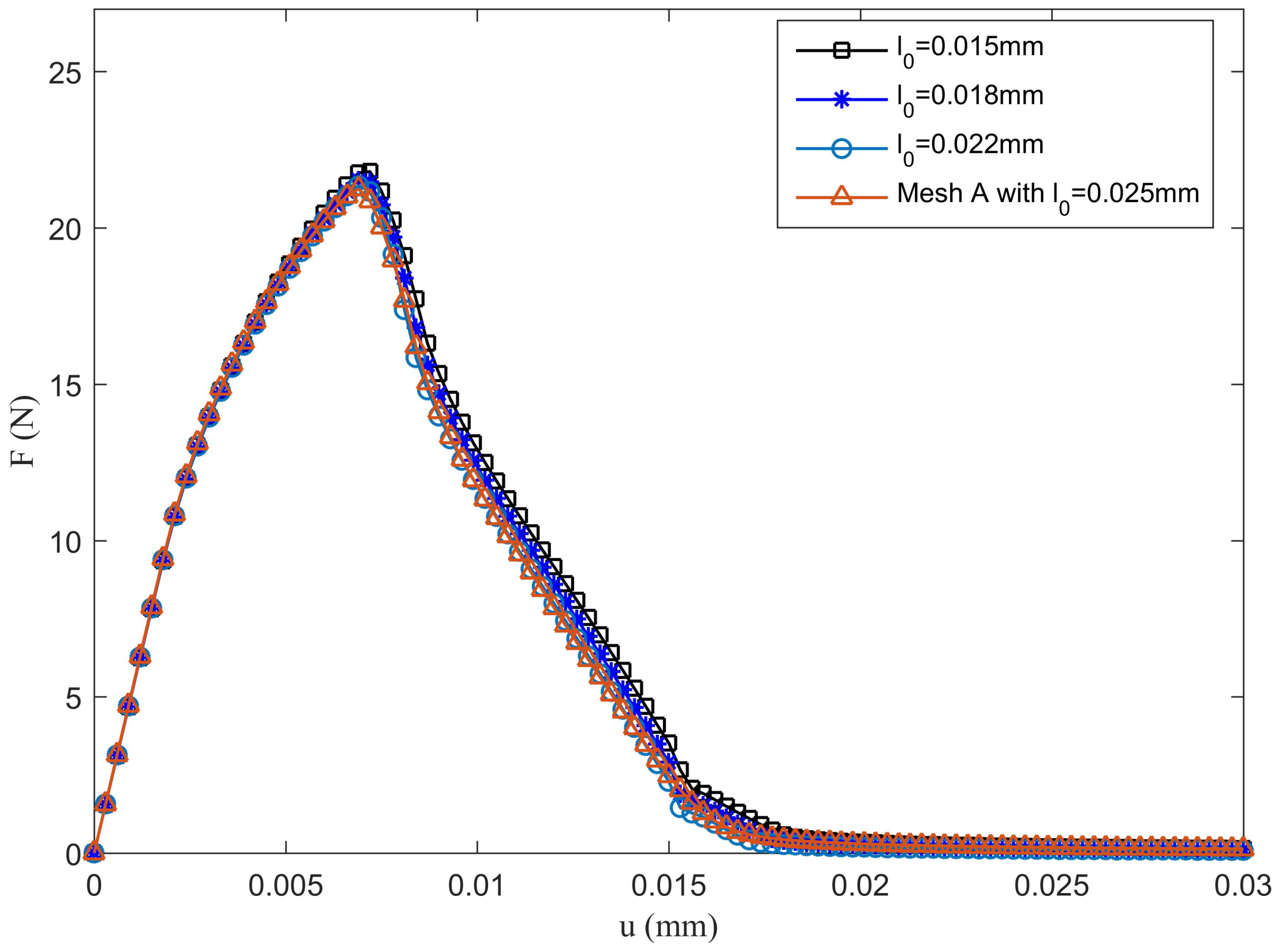




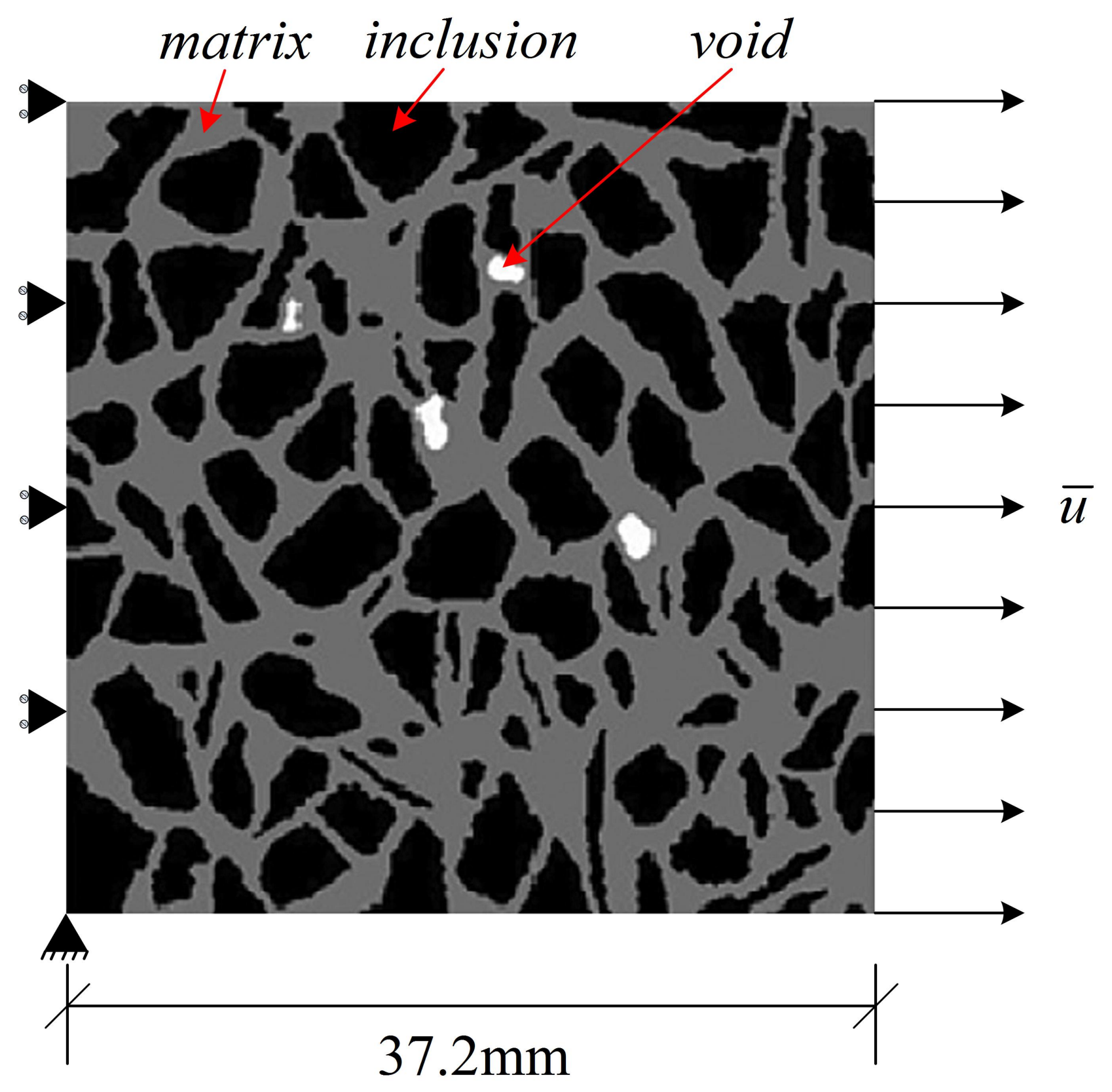



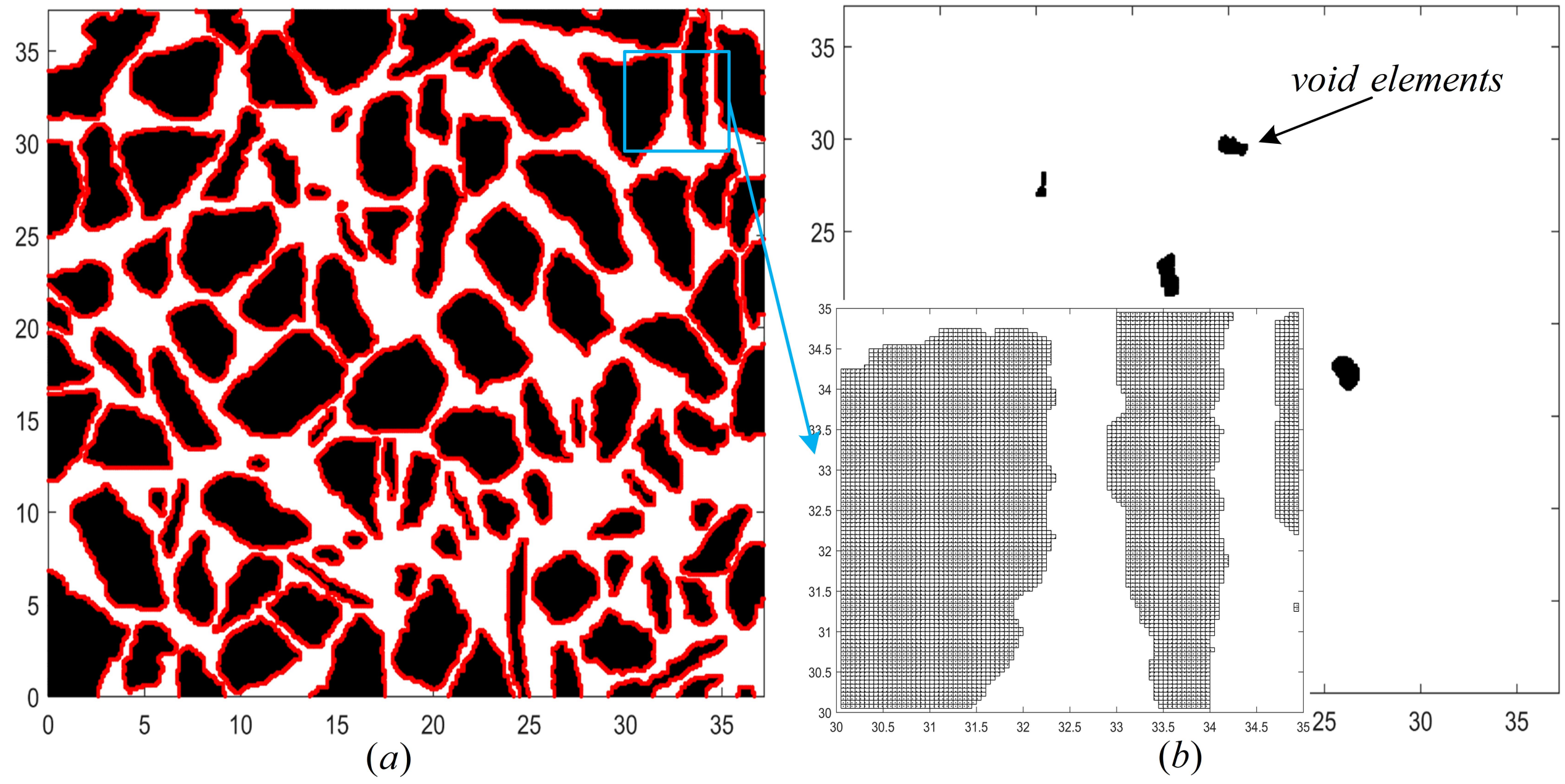


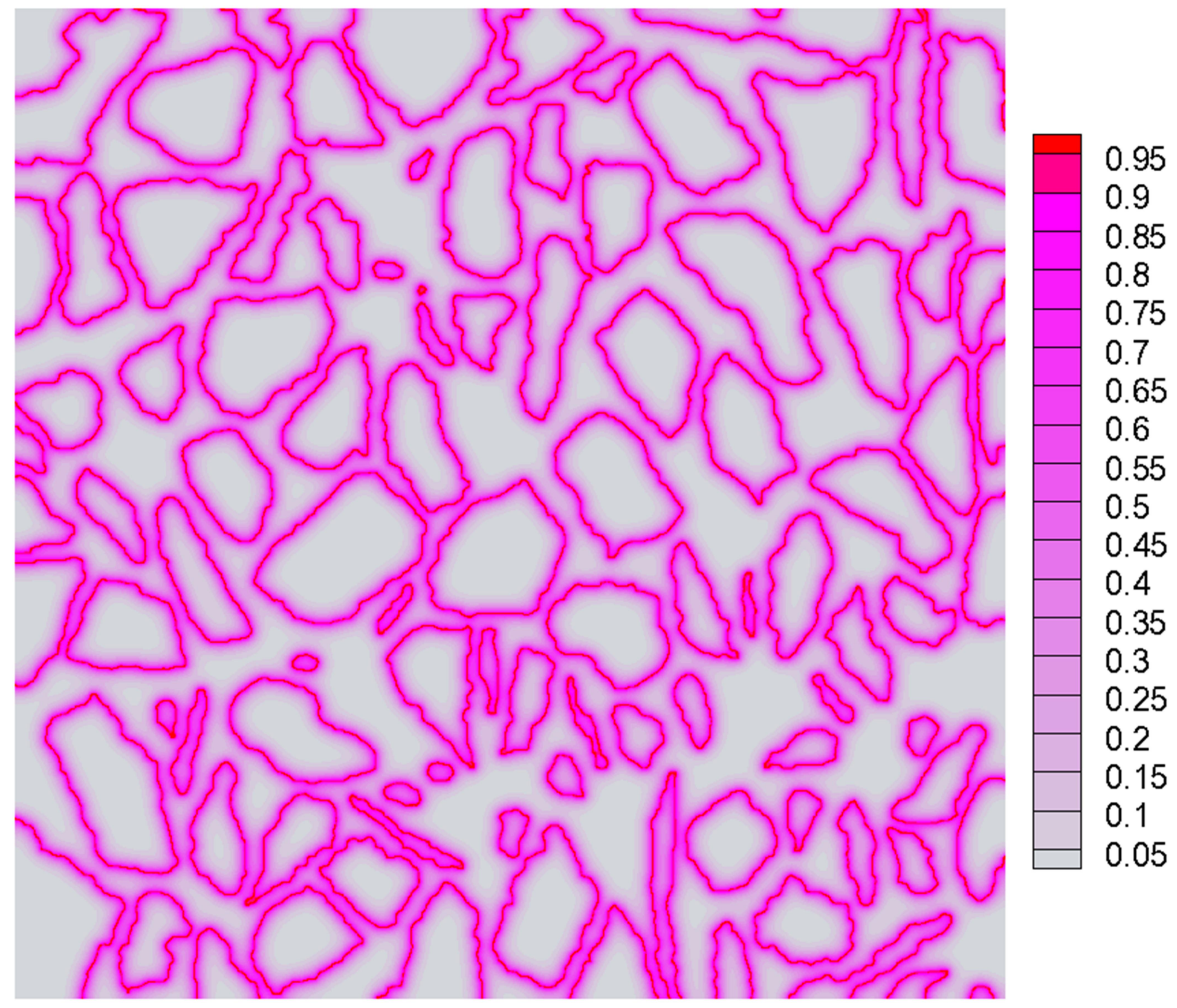




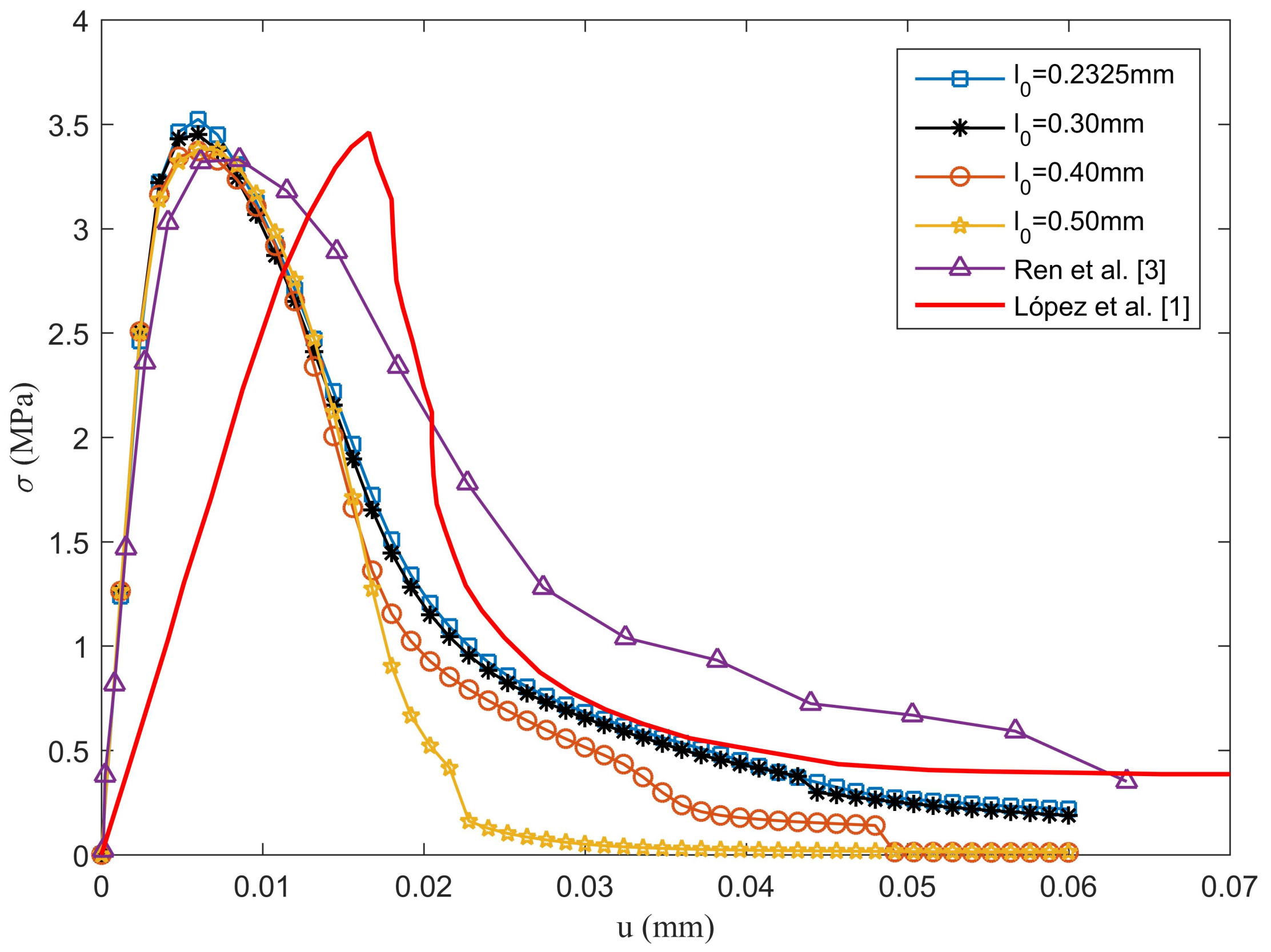




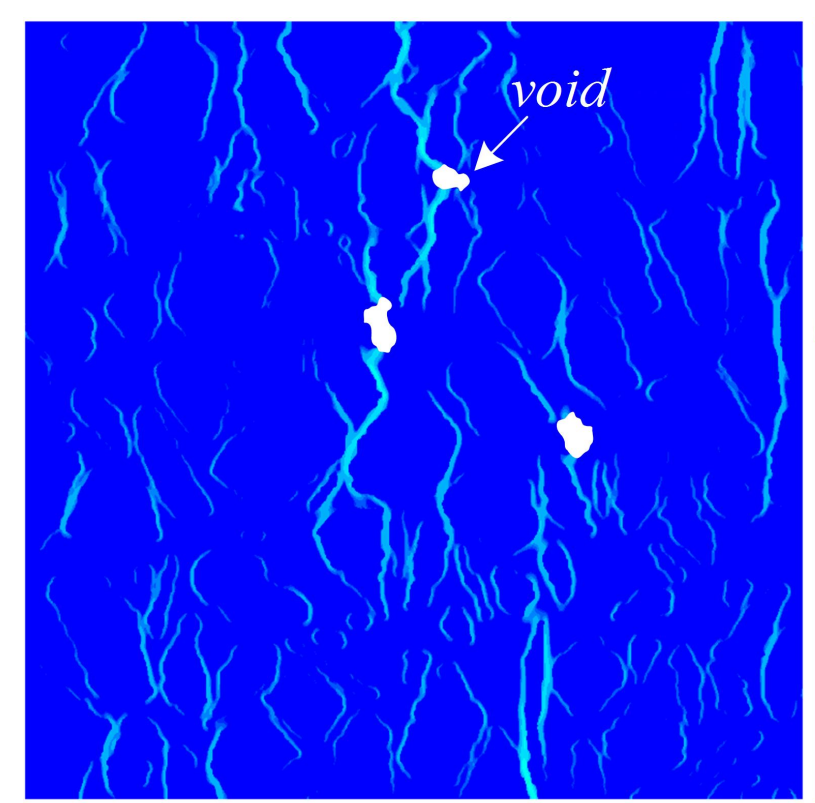

(a) Point A

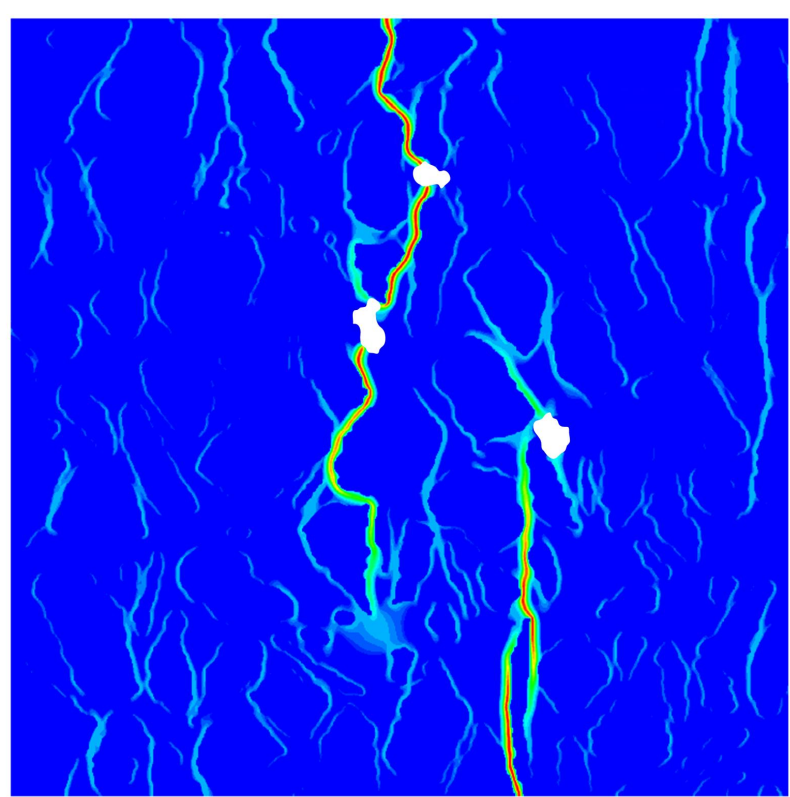

(b) Point $B$

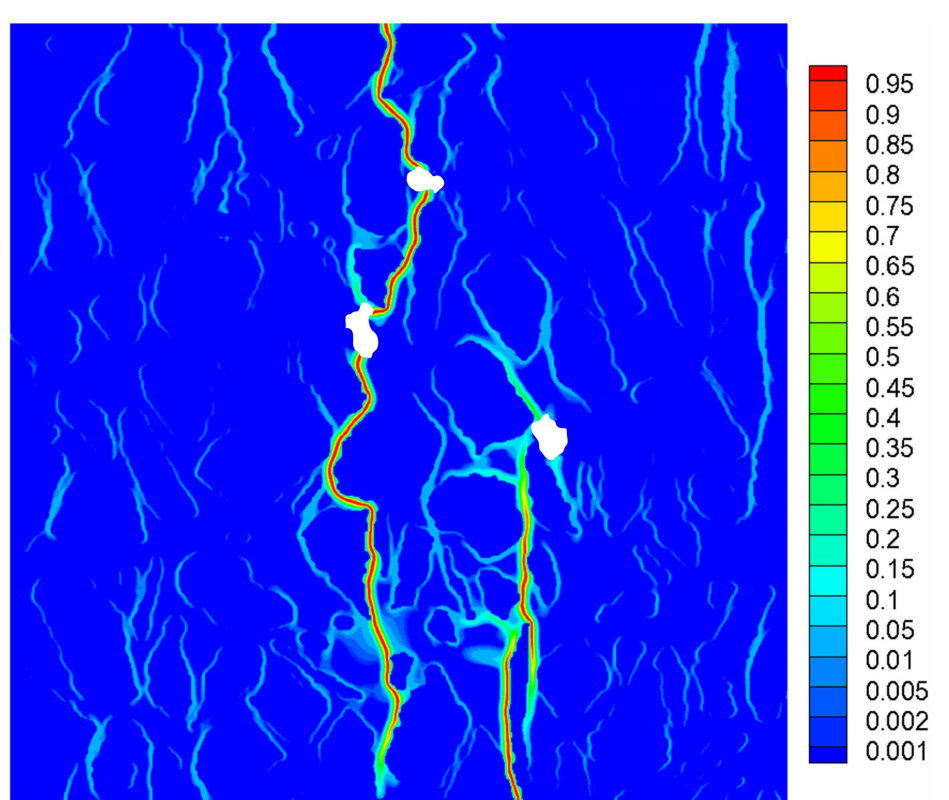

(c) Point $C$ 


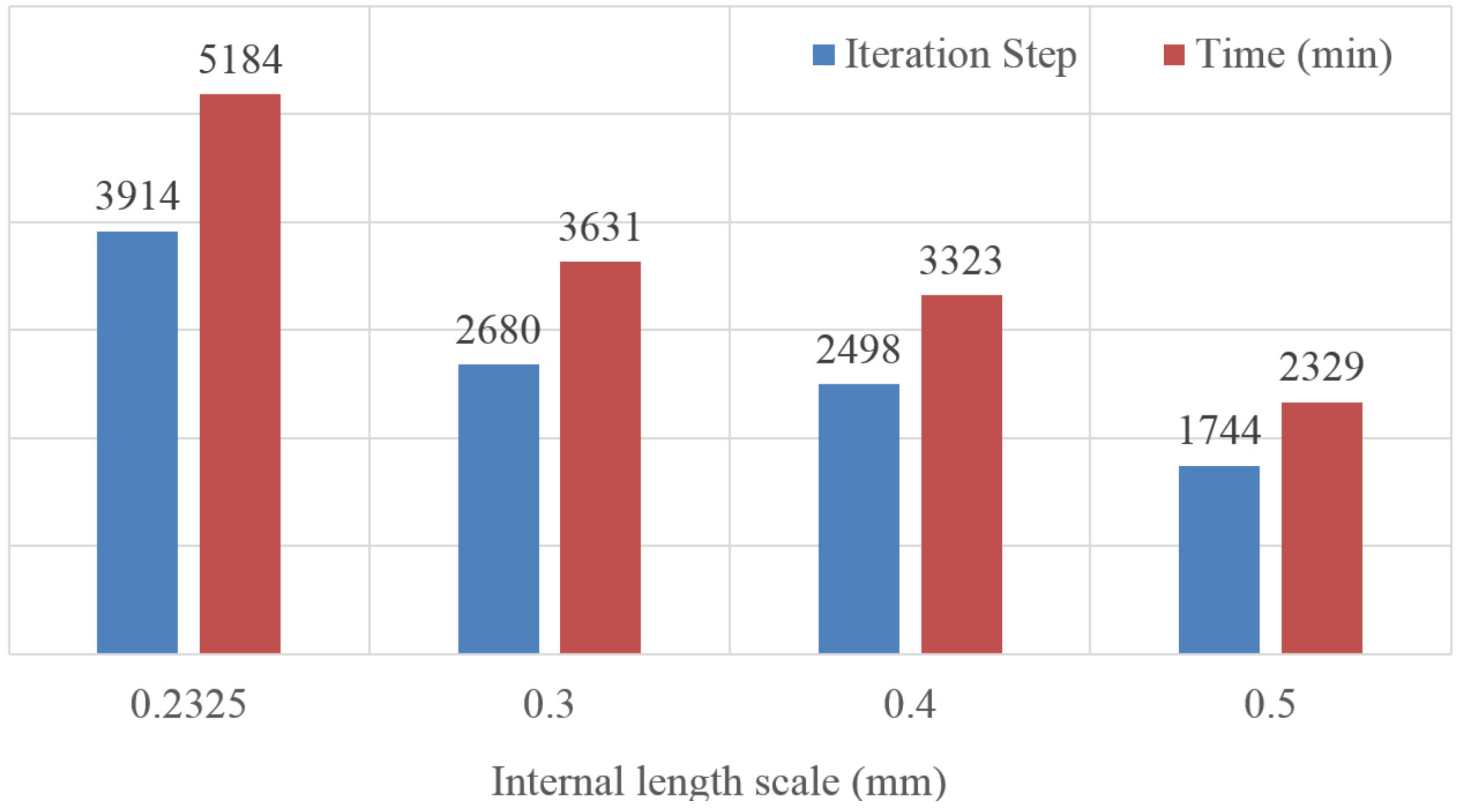




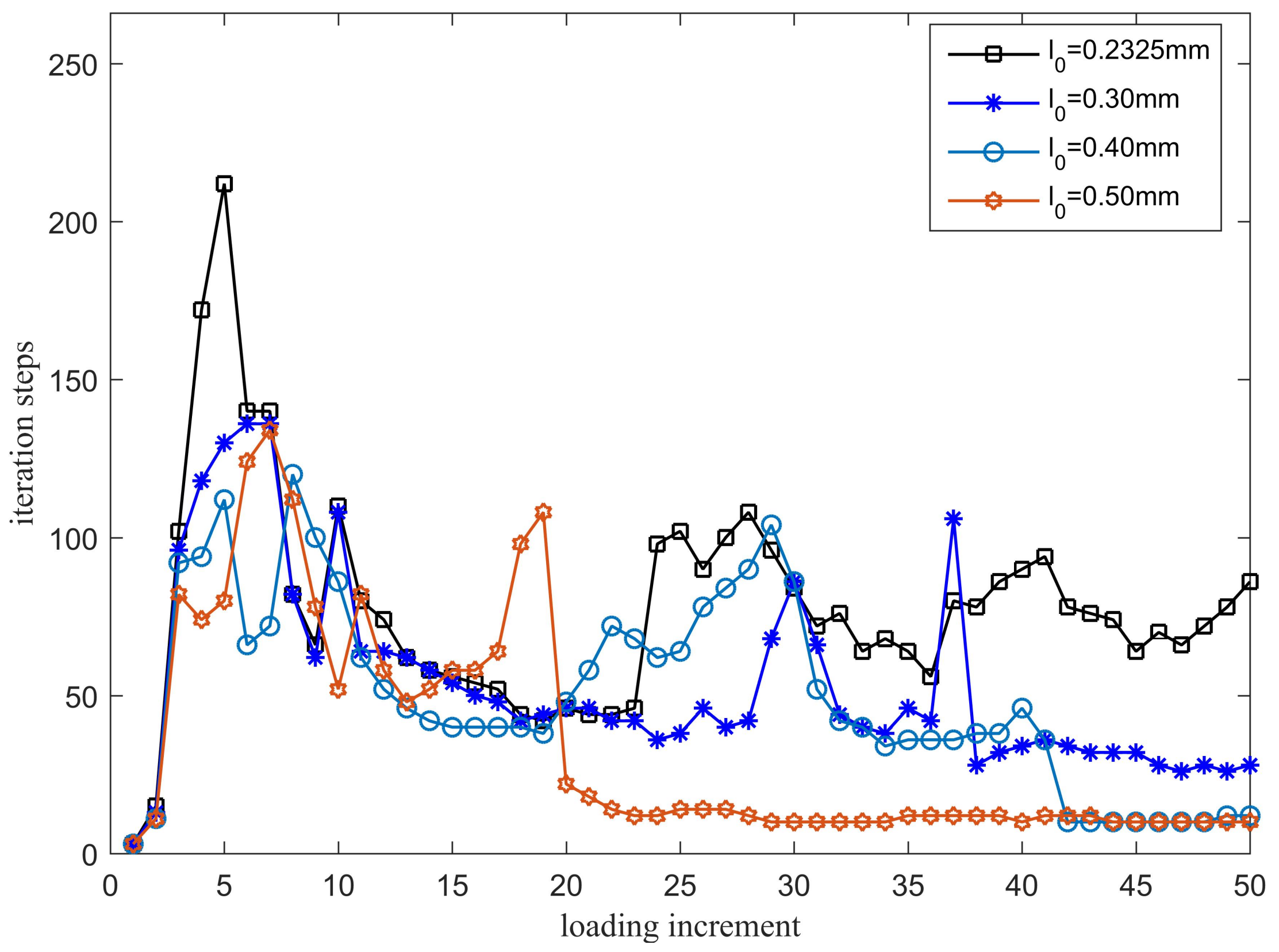

